\title{
Mahogunin-mediated $\alpha$-tubulin ubiquitination via noncanonical K6 linkage regulates microtubule stability and mitotic spindle orientation
}

\author{
D Srivastava ${ }^{1}$ and $O$ Chakrabarti ${ }^{*}, 1$
}

Mahogunin ring finger-1 (MGRN1) is a cytosolic ubiquitin ligase whose disruption or interaction with some isoforms of cytosolically exposed prion protein leads to spongiform neurodegeneration and also lack of which results in reduced embryonic viability due to mispatterning of the left-right (LR) axis during development. Here we demonstrate an interaction between the cytoskeletal protein $\alpha$-tubulin and MGRN1. In cultured cell systems, loss of the ubiquitin E3 ligase activity of MGRN1 results in spindle misorientation and decreased $\alpha$-tubulin polymerization, an effect also seen in primary cells. $\alpha$-Tubulin was posttranslationally modified by MGRN1 via noncanonical K6-linked polyubiquitination. This was significant because expression of catalytically inactive MGRN1 and/or ubiquitin mutant capable of only monoubiquitination resulted in similar mitotic spindle misorientation. The modulatory effect of MGRN1 was specific for $\alpha$-tubulin and similar changes could not be detected in $\beta$ - or $\gamma$-tubulin. However, catalytic inactivation of MGRN1 did not abrogate monoubiquitination of $\alpha$-tubulin, thus unraveling a unique dual mode of ubiquitination by an unknown E3 ligase and MGRN1. MGRN1-mediated $\alpha$-tubulin modification, and hence its stability, may highlight a key event in the LR patterning during embryogenesis.

Cell Death and Disease (2014) 5, e1064; doi:10.1038/cddis.2014.1; published online 20 February 2014

Subject Category: Neuroscience

Microtubules (MTs) are the fundamental structural elements of all cells that govern a myriad of cellular functions, including motility, maintenance of cell morphology, intracellular transport, mitosis and meiosis. A dynamic equilibrium exists between the assembly and disassembly of the MT network during the different phases of cell cycle. ${ }^{1}$ Proper assembly, positioning and orientation of the mitotic spindle are finely orchestrated by molecular players, like the dynein/dynactin complex that ensure correct spindle formation and generate the pulling forces required for proper cell division. ${ }^{2-4}$ These proteins are enriched at the spindle poles, and the disruption of a functional dynamin/dynactin motor protein complex impairs mitotic spindle morphology. 5,6

Recent reports indicate that Huntingtin (HTT) protein, mutations of which cause Huntington's disease (HD), localizes to spindle poles throughout mitosis. Downregulation of HTT in primary cells affects motor proteins and leads to spindle misorientation that translates to defects in murine neuronal progenitors and neuroblast precursors in Drosophila. $^{7}$
Furthermore, Parkin, a ubiquitin E3 ligase, ${ }^{8}$ is also a tubulinbinding protein. Parkin-tubulin interaction in human cell lines leads to increased ubiquitination and accelerated degradation of $\alpha$ - and $\beta$-tubulins. Point mutants of Parkin (K161N, T240R and $\mathrm{C} 431 \mathrm{H}$ ) identified in Parkinson's disease (PD) patients are incompetent in the E3 ligase activity toward tubulins. ${ }^{9}$

The role of post-translational modifications of tubulin, such as acetylation, methylation, detyrosination, glutamylation and glycation in MT formation and regulation, has been studied. ${ }^{10}$ However, our understanding of ubiquitination of tubulin is still premature and is primarily focused on regulating its degradation and turnover.

Recent reports indicate that BRCA1 (breast cancerassociated gene 1) protein binds to and ubiquitinates $\gamma$-tubulin that is crucial for maintaining appropriate centrosome number in cells. ${ }^{11-14}$ In addition, mutations in ubiquitin C-terminal hydrolase L1 (UCH L1), a cysteine hydrolase, ${ }^{15}$ expressed abundantly and exclusively in brain and reproductive tissues, ${ }^{16}$ are associated with PD and Alzheimer's diseases (AD). ${ }^{17}$ Studies show that besides being a deubiquitinating

\footnotetext{
${ }^{1}$ Biophysics and Structural Genomics Division, Saha Institute of Nuclear Physics, Kolkata, India

${ }^{*}$ Corresponding author: 0 Chakrabarti, Biophysics and Structural Genomics Division, Saha Institute of Nuclear Physics, Sector-1, Block-AF, Bidhannagar, Kolkata, West Bengal 700064, India. Tel: +91 332337 5345; Fax: +91 332337 4637; E-mail: oishee.chakrabarti@ saha.ac.in

Keywords: spindle misorientation; noncanonical polyubiquitination; tubulin polymerization

Abbreviations: Ab, antibody; AD, Alzheimer's diseases; BRCA1, breast cancer-associated gene 1; ${ }^{C t m} \mathrm{PrP}$, C-terminal transmembrane form of prion protein; cyPrP, cytosolic Prion; DMEM, Dulbecco's modified Eagle's medium; EGTA, ethylene glycol tetra acetic acid; ESCRT, endosomal sorting complex required for transport; FBS, fetal bovine serum; FCS, fetal calf serum; G2-M, Gap2-mitosis; GFP, green fluorescent protein; GTP, guanosine-5'-triphosphate; HA, hemagglutinin; HD, Huntington's disease; HEK293T, human embryonic kidney 293 transformed with T-antigen; He-Ne, helium-neon; HTT, Huntingtin; LR, left-right; MEF, mouse embryonic fibroblast; MGRN1, mahogunin ring finger-1; M phase, mitosis phase; MT, microtubule; Noc, nocodazole; NA, numerical aperture; p53, protein 53; P, pellet; PBS, phosphate buffer

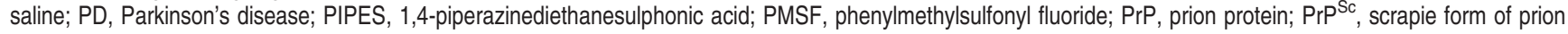
protein; Rad6, radiation gene 6; S, supernatant; SDS-PAGE, sodium dodecyl sulfate-polyacrylamide gel electrophoresis; siRNA, small interfering RNA; TPA, 12-Otetradecanoylphorbol 13-acetate; Tsg101, tumor suppressor gene 101; Tubgcp2, $\gamma$-tubulin-associated protein; Ub, ubiquitin; UbcH2, ubiquitin conjugating enzyme 2 (human); UCH L1, ubiquitin C-terminal hydrolase L1
}

Received 06.6.13; revised 19.12.13; accepted 19.12.13; Edited by GM Fimia 
enzyme, UCH L1 dimers have ubiquitin ligase activity in vitro and stabilize monoubiquitin in neurons. ${ }^{18-20}$

Prion diseases such as $H D, P D$ and $A D$ are late-onset neurodegenerative diseases. Prion protein ( $P r P)$, a highly conserved cell surface glycoprotein, is implicated in several of the prion diseases such as scrapie, bovine spongiform encephalopathy, Creutzfeldt-Jakob disease and Gerstmann-Straussler-Scheinker disease. Their pathogenesis is associated with the presence in brain of one or more abnormal isoforms of $\operatorname{PrP}$ (misfolded form of $\operatorname{PrP}\left(\mathrm{PrP}^{\mathrm{Sc}}\right.$ ), transmembrane isoform ( $\left.{ }^{\mathrm{Ctm}} \mathrm{PrP}\right)$ and cytoplasmic (cyPrP)) ${ }^{21}$ Although the mechanisms leading to disease are not fully understood, recent reports suggest that some of the diseasecausing isoforms of $\mathrm{PrP}$ can engage in atypical interactions with the cytosolic E3 ligase, mahogunin ring finger-1 (MGRN1). This results in inappropriate sequestration of MGRN1 in cell culture systems, and might contribute to lateonset neuronal dysfunction and disease, ${ }^{22}$ similar to homozygous loss of MGRN1 function leading to late-onset spongiform neurodegeneration in mice. ${ }^{23}$ However, the role played by MGRN1 in prion diseases caused by $\operatorname{PrP}^{\mathrm{Sc}}$ may not be as evident. $^{24}$

Separate studies indicate that although $\sim 50 \%$ of MGRN1null mutants in mice develop late-onset spongiform neurodegeneration, rest of the animals exhibit severe developmental defects (like congenital heart defects, abnormal craniofacial patterning and mispatterning of the left-right (LR) body axis) and increased mortality by weaning age. In addition, $<1 \%$ of animals also suffered from situs inversus (complete reversal of the left and right body axes). ${ }^{25,26}$ Hence, the effect of loss of function of MGRN1 on cell viability and ultimately on the development of animals seemed likely.

Here, we describe the serendipitous discovery of an interaction between MGRN1 and the cytoskeletal protein, $\alpha$-tubulin; and its effect on the axis of cell division during mitosis. Depletion of MGRN1 in cells affects ubiquitination of $\alpha$-tubulin, altering its polymerization state and not the protein turnover. Our analysis identifies a unique mode of ubiquitination of $\alpha$-tubulin by MGRN1 (utilizing K6 ubiquitin linkages) as a novel post-translation modification of this cytoskeletal protein, responsible for the formation and maintenance of the mitotic spindle fibers.

\section{Results}

Interaction of endogenous MGRN1 with $\alpha$-tubulin in mitotic cells. We observed in HeLa cells that the typical punctate/vesicular pattern of expression of endogenous MGRN1 with an obvious nuclear exclusion as seen in interphase cells gets altered and it now decorates mitotic spindles in dividing cells (Figure 1a). This pattern of close association of MGRN1 with mitotic apparatus could be detected through the different stages of mitosis ( $M$ phase) (Supplementary Figure S1A). Co-immunoprecipitation of synchronized cells indicates an interaction between MGRN1 and $\alpha$-tubulin (Figures $1 \mathrm{~b}$ and $\mathrm{c}$ and Supplementary Figure $\mathrm{S1B}$ ) and this requires the C-terminus of MGRN1 (Figure 1d and Supplementary Figure S1B). Co-immunocytochemistry also showed their pronounced colocalization on mitotic spindles (Figure 1e). Similar results were noted in mitotic
HEK293T (human embryonic kidney 293 transformed with T-antigen) cells (Figure 1f), suggesting a cell line-independent phenomenon. Furthermore, co-immunoprecipitation of melanocytes $^{27}$ (Supplementary Figure S1D) demonstrates an interaction between MGRN1 and $\alpha$-tubulin in melan a- 6 (control) cells when compared with melan md1-nc (Mgrn $1^{\text {md- }}$ ${ }^{n c}$ or Mgrn1 null) cells (Figures $1 \mathrm{~g}$ and $\mathrm{h}$ ). These observations led to the following plausible hypotheses - either association with MGRN1 regulates the turnover of $\alpha$-tubulin or MGRN1 ubiquitinates $\alpha$-tubulin to affect its function. However, an indirect interaction with the spindle assembly via the dynein/ dynactin complex seemed unlikely.

Catalytically inactive MGRN1 affects the axis of cell division. To understand the functional significance of the interaction between MGRN1 and $\alpha$-tubulin, cells were transiently transfected with full-length (referred to as MGRN1) or a catalytically inactive MGRN1, lacking the RING domain (MGRN1 $\Delta$ ), or with point mutations (C316DMGRN1, C299EMGRN1) in the same domain (Supplementary Figures S2A and B). Although HeLa cells transiently transfected with MGRN1 $\Delta \mathrm{R}$ completed $\mathrm{M}$ phase of cell cycle with a mitotic index comparable to those expressing either cytosolic green fluorescent protein (GFP) or MGRN1 (Supplementary Figure S1E), they displayed spindle misorientation. ${ }^{28}$ The spindle angle relative to the cell substrate adhesion plane (Supplementary Figure S2C) for MGRN1 RING mutant-expressing cells ( 55-70\%, depending on the construct) was $>10^{\circ}$, whereas control spindles were usually parallel to the substratum (Figures $2 a$ and $b$ and Supplementary Figures S2 D and E and S3A). Whereas control cells show an angle mostly around $10^{\circ}$, the average angle upon functional inactivation of MGRN1 was significantly higher (between $15^{\circ}$ and $30^{\circ}$ ), indicating aberrant spindle orientation relative to the substratum (Figures $2 \mathrm{~b}$ and $\mathrm{d}$ and Supplementary Figure S2E; right graphs). This phenotype was even more pronounced when MGRN1 was depleted from cells using small interfering RNA (siRNA) constructs (with knockdown efficiency of $\sim 75 \%$ ), where spindle tilt of $>10^{\circ}$ was seen in $\geq 80 \%$ of cells (Figures $2 \mathrm{c}$ and d). Such experiments with catalytically inactive MGRN1 were possible only with transient transfections as three attempts to establish stable cell lines failed (data not shown); on the contrary, we could easily establish stable cells expressing full-length MGRN1 (Supplementary Figure $\mathrm{S1C})$. Although no drastic effect of functionally inactive MGRN1 is obvious on the mitotic index or the completion of mitosis in the short term (Supplementary Figure S1E), the effect of spindle misorientation had a cumulative detrimental effect over multiple cycles of cell division.

We next examined the structural underpinnings of spindle misorientation induced by MGRN1 inactivation/depletion. The most notable defect was a significant loss and shortening of astral MTs that did not contact the cell cortex, a requirement for force generation during spindle orientation (Figures $2 \mathrm{e}-\mathrm{h}$ ).

We also evaluated the effect of MGRN1 inactivation on the motor protein, dyenin1, as it is important for spindle pole assembly and orientation. ${ }^{7,28}$ Immunocytochemistry showed a decrease in dynein staining at the poles when MGRN1 was depleted compared with the control cells (Figures $3 a$ and b). 
a

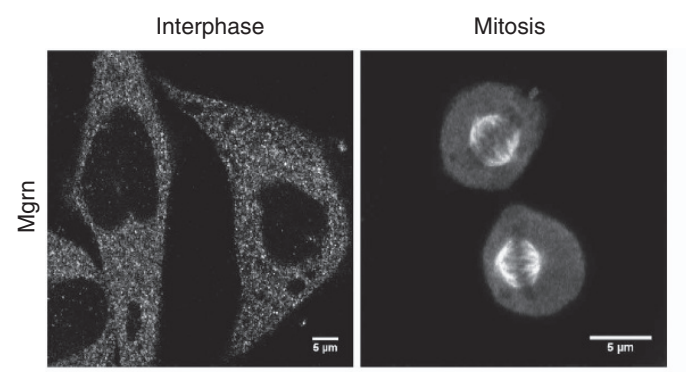

b

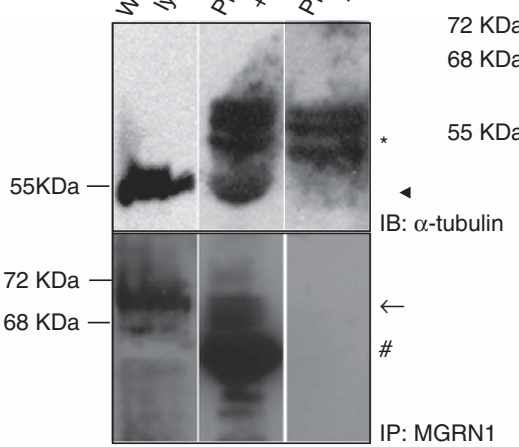

C

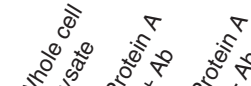

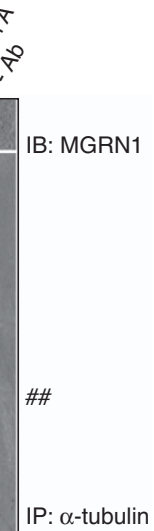

d
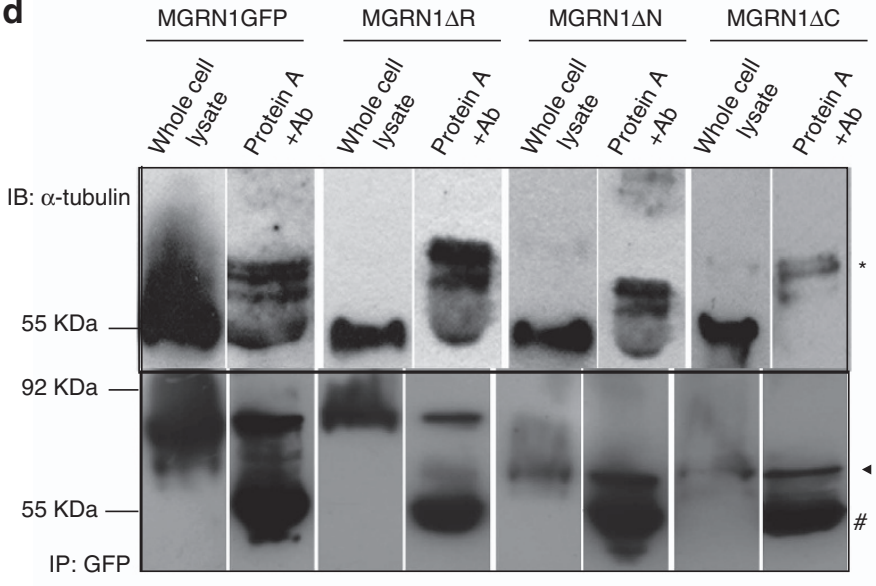

g
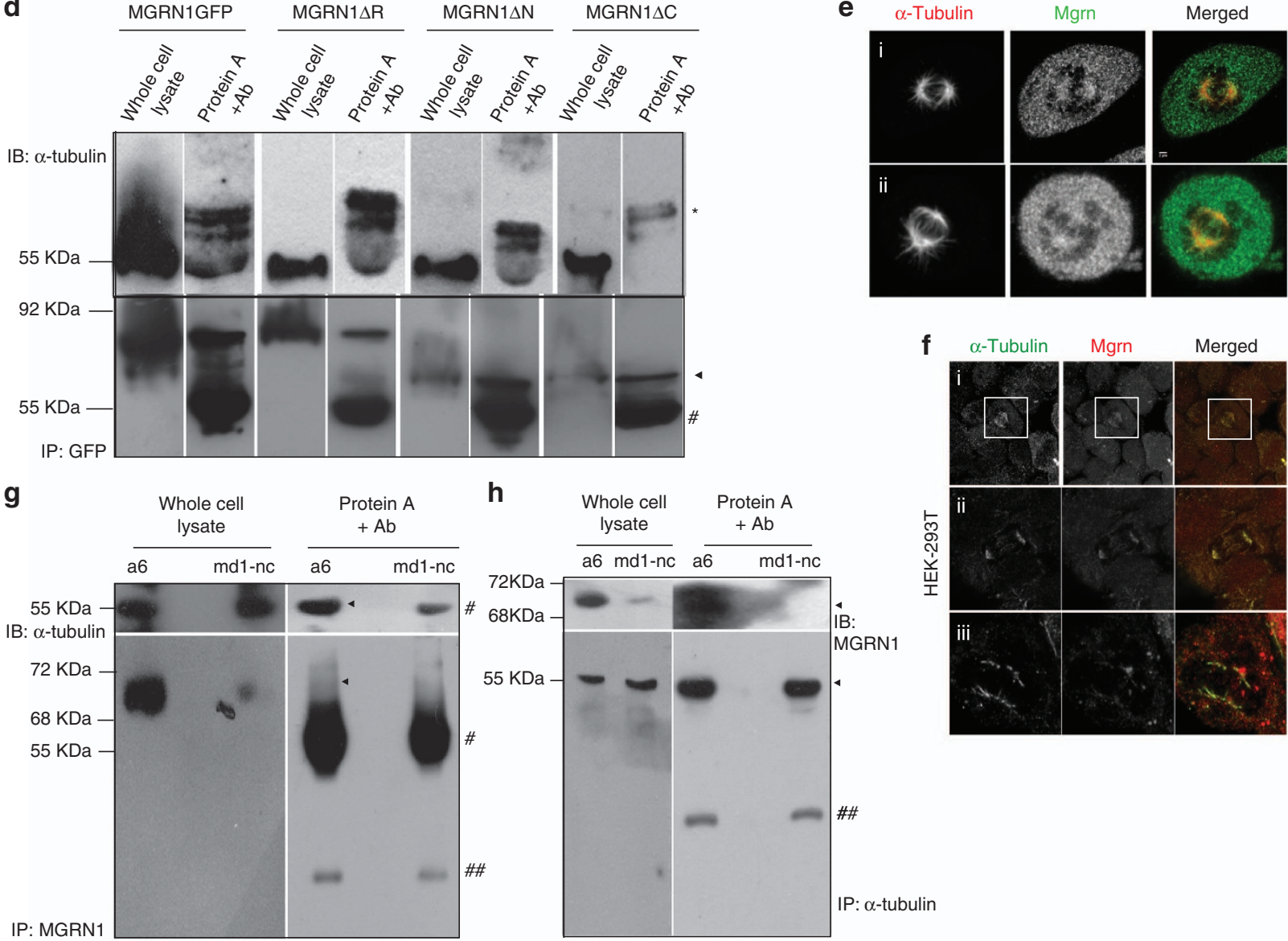

f
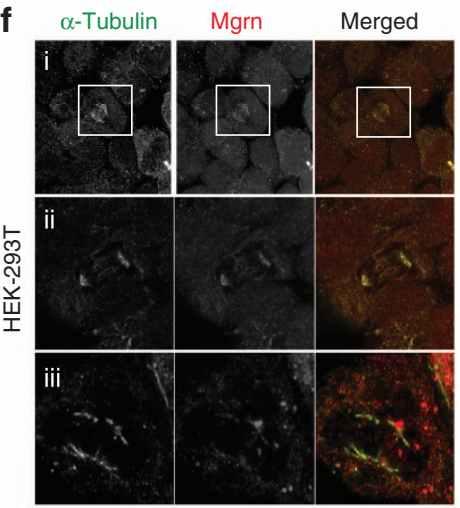

Figure 1 Mahogunin interacts with $\alpha$-tubulin. (a) Unsynchronized HeLa cells immunostained for endogenous MGRN1 show distinct nuclear exclusion during interphase but decorate the mitotic spindles in dividing cells. (b) Synchronized population of HeLa cells, enriched for mitotic cells, were lysed and immunoprecipitated with anti-MGRN1 antibody. Western blots analysis of this with anti- $\alpha$-tubulin antibody shows co-immunoprecipitation of $\alpha$-tubulin with MGRN1. 4 Marks $\alpha$-tubulin; $\leftarrow$ marks MGRN1; Ab, antibody; * Indicates nonspecific band; and ${ }^{\#}$ indicates immunoglobulin G (IgG) heavy chain. (c) Synchronized HeLa cells were lysed and immunoprecipitated with anti$\alpha$-tubulin antibody. Immunoblotting with anti-MGRN1 antibody shows co-immunoprecipitation between the two proteins. \#\# Indicates IgG light chain. (d) HeLa cells transiently transfected with the indicated GFP-tagged MGRN1 constructs were synchronized, lysed and immunoprecipitated with anti-GFP antibody. Western blots analysis of this with anti- $\alpha$-tubulin antibody shows co-immunoprecipitation of $\alpha$-tubulin with MGRN1, MGRN1 $\Delta R$ and MGRN1 $\Delta$ N but not MGRN1 $\Delta \mathrm{C}$ (top panel). Control blot immunoprecipitated with anti-GFP antibody to show expression of different MGRN1 constructs (bottom panel). (e) Synchronized population of HeLa cells immunostained for MGRN1 and $\alpha$-tubulin show colocalization between the two proteins on the mitotic apparatus. The channels for acquiring the images are indicated. Two representative cells (i) and (ii) are shown. (f) Colocalization between MGRN1 and $\alpha$-tubulin is detected in mitotic HEK-293T cells. The channels for acquiring the images are indicated. Three representative cells (i), (ii) and (iii) are shown. (g) Asynchronous a-6 and md1-nc melanocyte cells were lysed and immunoprecipitated with anti-MGRN1 antibody. Western blots analysis of this with anti$\alpha$-tubulin antibody shows co-immunoprecipitation of $\alpha$-tubulin with MGRN1 in case of melan a-6 but in the melan md1-nc cells. It should be noted that as IgG heavy chain also migrates at $\sim 55 \mathrm{KDa}$, a band (of lower intensity) is detected even in melan md1-nc cells. Marks MGRN1.(h) Asynchronous a-6 and md1-nc melanocyte cells were lysed and immunoprecipitated with anti- $\alpha$-tubulin antibody. Immunoblotting with anti-MGRN1 antibody shows co-immunoprecipitation between the two proteins in case of control melanocyte cell line (melan a-6) only but not in MGRN1-null cell line (melan md1-nc) 
However, the spread and pattern of distribution of dynein at and around the spindle poles remained very similar in both the cell populations (Figure $3 c$ ). These results imply that the decrease in detectable dyenin at the spindle pole might be due to its reduced association with polymerized $\alpha$-tubulin when MGRN1 is functionally inactive, rather than a direct effect on the inhibition in dynein/dynactin-dependent transport. ${ }^{7,29}$ a
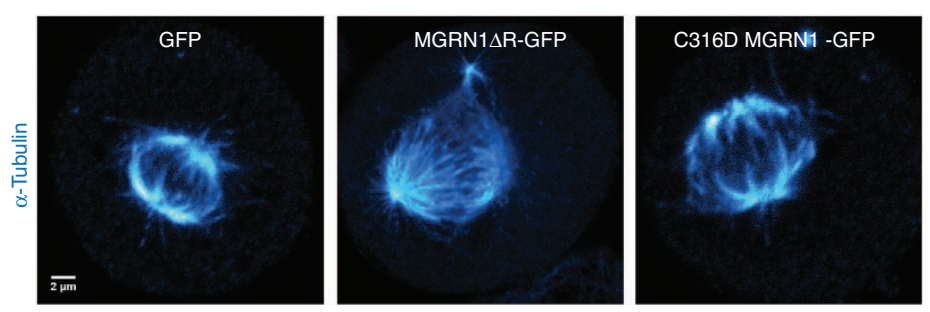

c
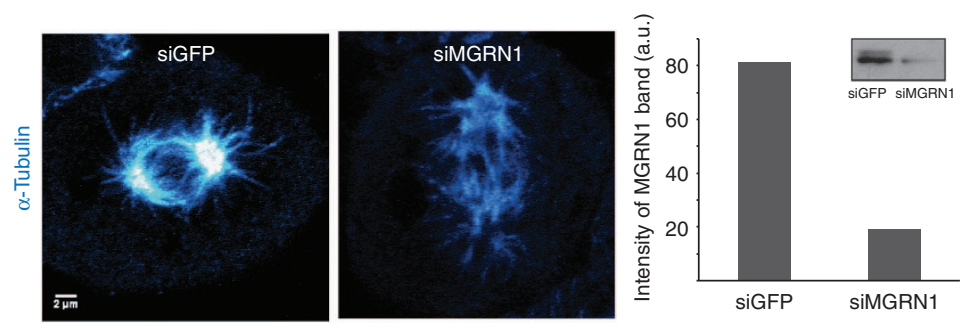

e
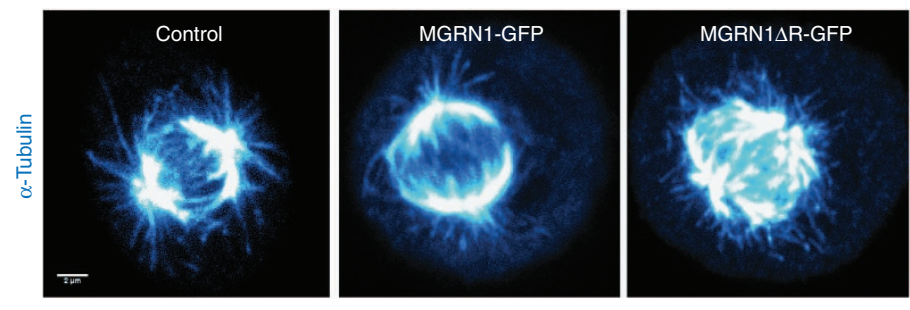

g
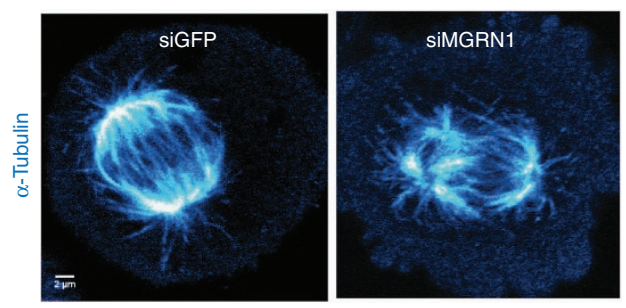
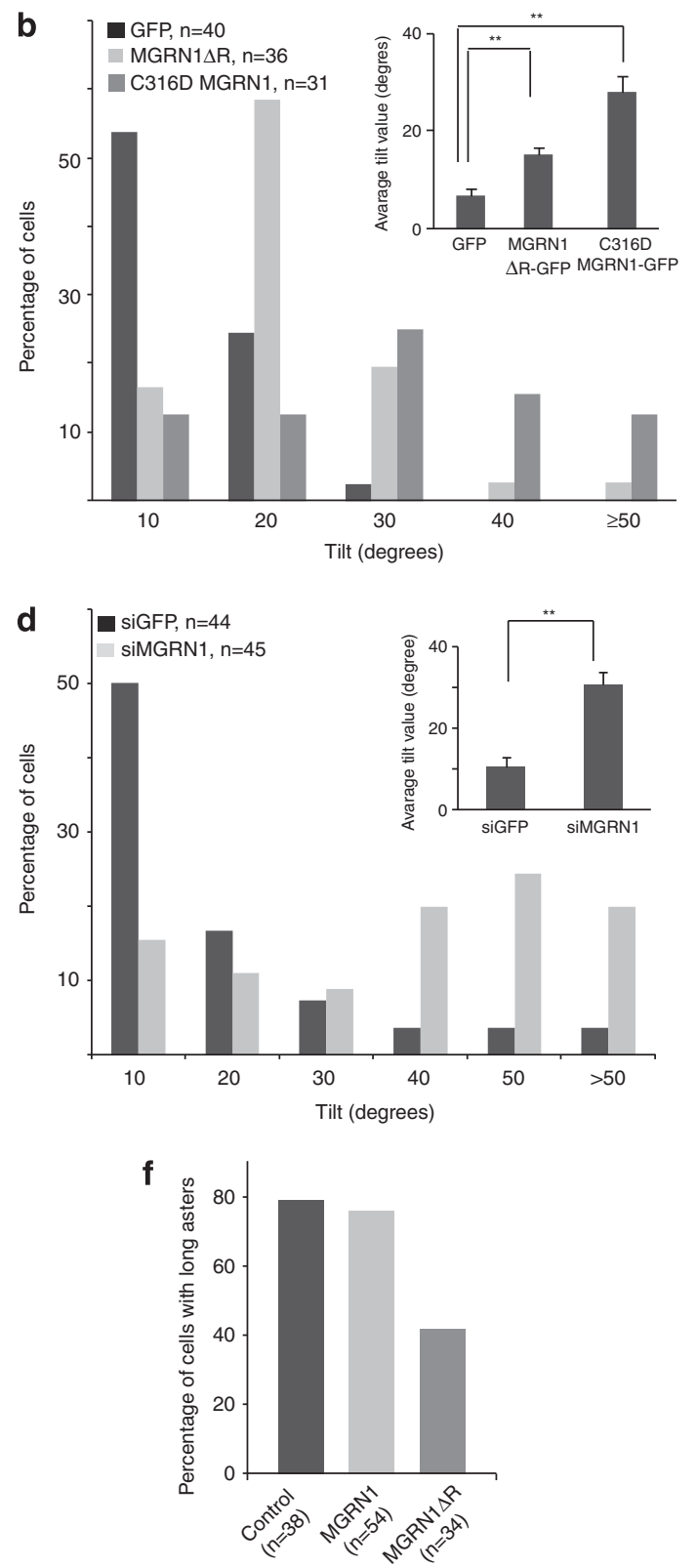

h

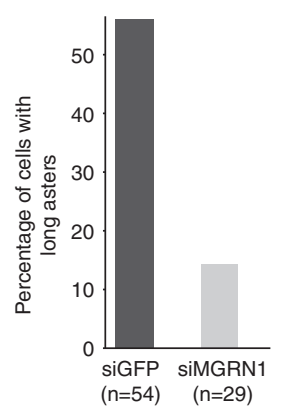


MGRN1 affects $\alpha$-tubulin polymerization. As mitotic spindles and asters are dynamic microtubule-based structures, we depolymerized microtubules with nocodazole (Noc) and followed their regrowth after washout of the drug in HeLa cells transiently expressing MGRN1, MGRN1 $\Delta \mathrm{R}$ or MGRN1 C316D at various time points (Figures $3 d$ and $e$ and Supplementary Figure S3B). Peripheral MT clusters, representing ectopic poles, were marginally more in number (1.51.7 times, across time points) upon functional inactivation of MGRN1 when compared with the control cells (Figure 3e). This difference remained very similar over the entire time period of the experiment, indirectly pointing to the fact that motor proteins do not directly govern this phenotype.

Furthermore, to biochemically establish the interaction between MGRN1 and polymerized MTs, co-sedimentation assay was performed using synchronized mitosis-enriched HeLa cell lysates. Results indicated that MGRN1 co-pelleted with taxol-stabilized MTs and not with Noc (negative control) (Figure 4a).

Finally, to check the status of endogenous microtubule polymerization in unsynchronized population of cells, HeLa cells and wild-type mouse embryonic fibroblasts (MEFs; , primary cells) were transiently transfected with the indicated MGRN1 constructs. At $24 \mathrm{~h}$ after transfection, the cells were lysed, $1 / 15$ th of this was retained for analysis as total lysate control and the rest was separated into soluble (supernatant (S)) and insoluble (pellet $(P)$ ) fractions and biochemically analyzed to detect any change in $\alpha$-tubulin polymerization among the samples (Figures $4 \mathrm{~b}-\mathrm{g}$ ). Melanocytes (melan a-6 and melan md1-nc) cells were grown for $24 \mathrm{~h}$, similarly fractionated and analyzed by western blots for the levels of $\alpha$-tubulin. Our results showed that although the S/P ratio is $\sim 1$ in control HeLa cells expressing cytosolic GFP or MGRN1; the ratio is $>2.5$ in cells overexpressing catalytically inactive MGRN1 (MGRN1 $\Delta \mathrm{R}$ or MGRN1316D), hence indicating a decrease in the polymerized form of $\alpha$-tubulin (Figures $4 b$ and c). Similarly, the S/P ratio was $>3$ in the presence of MGRN1 siRNA as compared with control $(\mathrm{S} / \mathrm{P} \approx 2$; Figures $4 \mathrm{~d}$ and $\mathrm{e})$. The most pronounced change was observed with wild-type MEFs in a similar experiment - with S/P ratio of $\sim 3.6$ upon overexpression of MGRN1 $\Delta R$ compared with the MGRN1 control having S/P ratio of $\sim 1.4$ (Figures $4 f$ and $\mathrm{g}$ ). These samples when analyzed for changes in $\beta$-tubulin polymerization failed to elicit similar differences, irrespective of the functional status of MGRN1 (Figures 4j-m). In melanocytes, whereas the S/P ratio was $\sim 1.9$ for melan a-6, it was $\sim 3$ for melan md1-nc cells (Figures $4 \mathrm{~h}$ and i).

Furthermore, in Noc washout experiments with HeLa cells expressing MGRN1, MGRN1 $\mathrm{R}$ or C316DMGRN1 to study the MT network, the interphase cells showed that catalytic inactivation of MGRN1 distinctly compromised formation of a polymerized mesh - the MGRN1 $\mathrm{R}$ - or C316DMGRN1expressing cells lag behind the MGRN1 cells by $\sim 30 \mathrm{~min}$ (Figure 5a and Supplementary Figure S4). siRNA-mediated depletion of MGRN1 also severely affected microtubule reassembly (Figure $5 \mathrm{~b}$ ). Wild-type MEFs subjected to similar microtubule regrowth assay after Noc treatment over a period of 10 and 15 min show a very punctate $\alpha$-tubulin expression pattern in MGRN1 $\Delta \mathrm{R}$ cells as compared with a well-polymerized $\alpha$-tubulin network and MT cluster nucleation centers in MGRN1 cells (Supplementary Figure S5B). Immunocytochemistry showed that like HeLa cells, MEFs also endogenously express MGRN1 (Supplementary Figure S5A).

Cumulatively, these results indicate a decrease in MT polymerization when the catalytic activity of MGRN1 is compromised in a cancer-derived cell line as well as primary cells.

MGRN1 does not affect $\gamma$-tubulin polymerization. Immunocytochemistry of HeLa cells for the endogenous levels of $\gamma$-tubulin and MGRN1 on mitotic cells showed that although $\gamma$-tubulin was present only on the spindle poles, MGRN1 decorates the entire spindle apparatus (Figure 6a). We next checked whether $\gamma$-tubulin was affected by MGRN1 depletion (Figures $6 \mathrm{~b}$ and $\mathrm{c}$ ). The expression of $\gamma$-tubulin, its intensity and spread at the poles remain independent of the presence of functional MGRN1, implying that in our system the formation and orientation of spindle poles represent two discrete events.

Furthermore, to check the status of endogenous $\gamma$-tubulin polymerization, HeLa cells expressing MGRN1, MGRN1 $\Delta R$,

\footnotetext{
Figure 2 Functional depletion of MGRN1 results in tilt in the axis of cell division. (a) HeLa cells transiently transfected with the indicated constructs were synchronized and immunostained for $\alpha$-tubulin. Mitotic cells positive for GFP expression were imaged by taking $z$-sections. The images are representative mid-sections (of the $z$-stacks) from at least 30 cells for each of the constructs. A larger proportion of cells expressing catalytically inactive MGRN1 have angle of tilt $\geq 10^{\circ}$ as compared with the control. The lookup table (LUT) images are represented to efficiently locate the spindle poles. (b) Histogram plotting the distribution and average of the angles of tilt for cells transfected with cytosolic GFP (control), MGRN1 $\triangle$ R-GFP or C316DMGRN1-GFP. Over 30 cells analyzed as in (a) are represented for each of the constructs. Graph shows results from three independent experiments. ${ }^{* \star} P \leq 0.001$, using Student's $t$-test. Error bars, S.E.M. (c) HeLa cells treated with MGRN1 siRNAs or irrelevant siRNAs (GFP siRNAs) were imaged and analyzed as in (a). Note that MGRN1 knockdown closely phenocopies MGRN1 $\Delta$ R expression in affecting the orientation of the axis of cell division. The immunoblot and graph shows a knockdown efficiency of $\sim 75 \%$. LUT images are represented to efficiently locate the spindle poles. (d) Histogram plotting the distribution and average of the angles of tilt for cells treated with MGRN1 siRNAs or irrelevant siRNAs (GFP siRNAs). Over 40 cells analyzed as in (c) are represented for each of the siRNA treatments. Graph shows results from three independent experiments. ${ }^{*} P \leq 0.001$, using Student's $t$-test. Error bars, S.E.M. (e) HeLa cells either untransfected (control) or transiently transfected with the indicated constructs were synchronized and immunostained for $\alpha$-tubulin. Mitotic cells positive for GFP expression were imaged by taking $z$-sections. The images are representative $z$-projections of at least 30 cells for each of the constructs. The images were acquired with high detector gains and saturating pixels to enable efficient visualization of the thin aster rays and the limiting boundary of the cells. Note shortening of aster rays in MGRN1 $\Delta$ R-GFP in comparison with either MGRN1-GFP or control cells. The cells analyzed were from the same experiments as in (a). LUT images are represented to efficiently locate the spindle poles and thin aster rays. (f) Histogram plotting the percentage of cells with long asters (ratio of the aster length to the cell diameter $>0.15$ ) upon expression of the constructs as indicated on the $X$ axis. Over 30 cells were analyzed for this. Graph shows results from three independent experiments. (g) HeLa cells treated with MGRN1 siRNAs or irrelevant siRNAs (GFP siRNAs) were imaged and analyzed as in (f). The images were acquired with high detector gains and saturating pixels to enable efficient visualization of the thin aster rays and the limiting boundary of the cells. Note that MGRN1 knockdown closely phenocopies MGRN1 $\Delta$ R expression in length of the aster rays. The cells analyzed were from the same experiments as in (c). LUT images are represented to efficiently locate the spindle poles and thin aster rays. (h) Histogram similar to (f) was plotted to check the effect of MGRN1 knockdown on aster length. Note a marked decrease in aster length in the absence of MGRN1. Approximately 30 cells were analyzed. Graph shows results from three independent experiments
} 
a
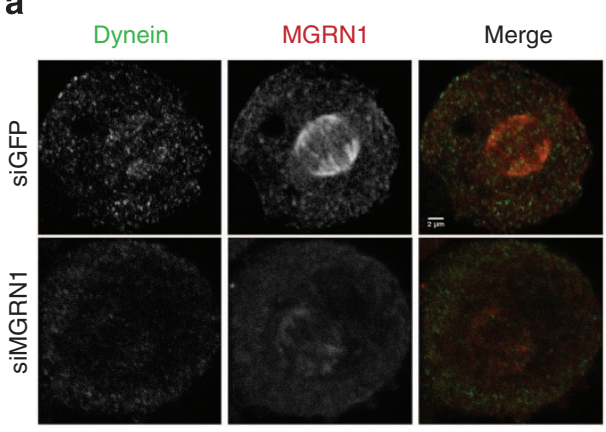

b

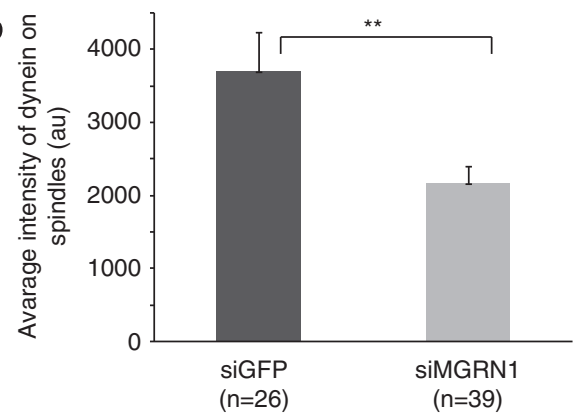

C

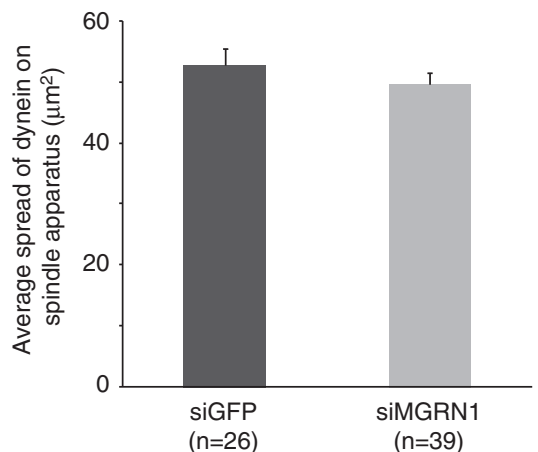

d

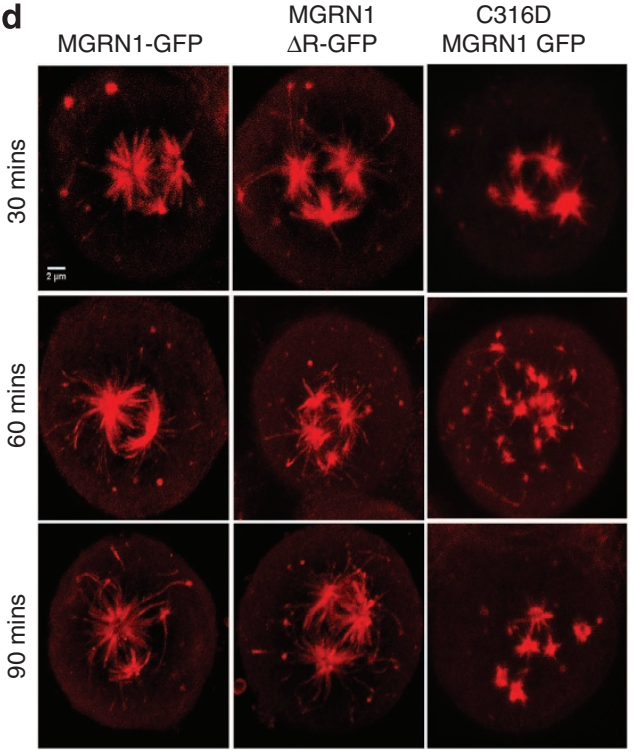

$\alpha-$ Tubulin

e

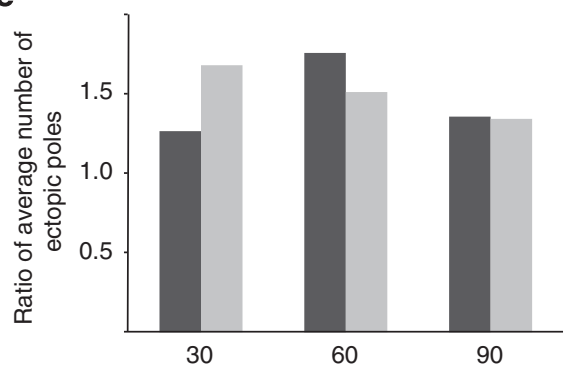

Revival time after removal of nocodazole (mins)

Ratio in MGRN1 $\triangle R$ GFP to MGRN1 GFP

Ratio in C316D MGRN1 GFP to MGRN1 GFP

Figure 3 Interaction of MGRN1 with $\alpha$-tubulin is independent of motor protein dynein. (a) HeLa cells treated with MGRN1 siRNAs or irrelevant siRNAs (GFP siRNAs) were co-immunostained for dynein intermediate chain (dynein) and MGRN1; mitotic cells were imaged. Note reduced intensity of detectable dynein with MGRN1 knockdown when compared with the control cells. Both the cell populations were imaged with identical microscope settings. The channels for acquiring the images are indicated. (b) Histogram plotting the average intensity of dynein on the spindles (in arbitrary units) in cells imaged in (a). Note a significant decrease in spindle dynein intensity with MGRN1 siRNA treatment; ${ }^{* *} P \leq 0.01$, using Student's $t$-test. Error bars, S.E.M. Graph shows results from three independent experiments. (c) Same samples as in (a) were analyzed and plotted for the average spread of dynein staining at the mitotic apparatus. This difference in the spread between the cells was not significant $(P \geq 0.1)$ using Student's $t$-test. Error bars, S.E.M. Graph shows results from three independent experiments. (d) HeLa cells transiently transfected with the indicated constructs were treated with nocodazole and allowed to recover for various time points $(30,60$ and $90 \mathrm{~min})$. The cells were immunostained for $\alpha$-tubulin. Note an increase in ectopic poles with the functional depletion of MGRN1. The channel for acquiring the images is indicated. (e) Cells imaged in (d) were analyzed for the total number of ectopic poles. The ratio of the average number of ectopic poles between MGRN1 $\triangle R / C 316$ DMGRN1 and MGRN1 was plotted for each of the time points. Note that this ratio remains similar over time. Graph shows results from three independent experiments

C316DMGRN1 or depleted for the MGRN1 expression were lysed, separated into $S$ and $P$ fractions and biochemically analyzed (Figures $6 \mathrm{~d}-\mathrm{g}$ ). Again, we could not detect any significant difference in the $S / P$ ratios between the various experimental (with inactive MGRN1) and control samples.

These results again imply that MGRN1 dysfunction does not affect $\gamma$-tubulin polymerization, its localization or distribution and hence, in turn, does not compromise spindle pole formation while still severely altering the pole orientation.

MGRN1 affects $\alpha$-tubulin polymerization by its polyubiquitination. Tsg101 (tumor suppressor gene 101), a key component of the ESCRT-I (endosomal sorting complex required for transport-I), is the only known physiological substrate that is monoubiquitinated by MGRN1 at multiple sites. ${ }^{30}$ Like other ubiquitin E3 ligases, MGRN1 is capable of generating a polyubiquitin ladder and also its autoubiquitination in the presence of a functional RING domain. ${ }^{23} \mathrm{We}$ therefore tested whether in vivo $\alpha$-tubulin was ubiquitinated by MGRN1. Lysates derived from HeLa cells co-transfected with either MGRN1 or MGRN1 $\Delta R$ and separated into $S$ and $P$ fractions were immunoprecipitated with $\alpha$-tubulin antibody (Ab) and immunoblotted for endogenous ubiquitin (Figure $7 \mathrm{~b}$ ). Detection of stronger signal in the $\mathrm{P}$ fractions indicates the prevalence of ubiquitinated species in them and 
also strengthens the argument for the involvement of this modification in the polymerization of $\alpha$-tubulin. Detectable level of ubiquitinated $\alpha$-tubulin is less in the $P$ fraction of MGRN1 $\Delta R$ as compared with MGRN1.

We next asked whether this was a mono- or polyubiquitination event. To address this, various hemagglutinin (HA)tagged constructs of different ubiquitin species - wild-type ubiquitin (Ub), K0, K6, K11, K29, K48 or K63 (ubiquitin mutants with arginine substitutions at all lysine residues except the ones indicated) - were used (Figure 7a). ${ }^{31-33}$

Lysates derived from HeLa cells co-transfected with either MGRN1 or MGRN1 $\Delta \mathrm{R}$ along with the various ubiquitin constructs (Figure 7c) showed that $\alpha$-tubulin underwent polyubiquitination in the presence of MGRN1 and Ub (lane 1, as evidenced by the detection of a polyubiquitin ladder); however, this was abrogated in the presence of MGRN1 $\Delta R$ and Ub (lane 2), implying that the inhibition of the MGRN1 catalytic activity affects $\alpha$-tubulin ubiquitination. As MGRN1 is known to multi-monoubiquitinate $\mathrm{Tsg} 101,{ }^{30}$ it was obvious to check the effect of $\mathrm{KO}$ in a similar assay. However, unlike with Ub, we could not detect any multi-monoubiquitination in the presence of MGRN1 and K0 (lane 3) even on a darker exposure. Multi-monoubiquitination was, however, clearly evident in lane 4, having MGRN1 $1 \mathrm{R}$ and $\mathrm{KO}$; this could probably mean than in the absence of a catalytically active MGRN1, there was enforced multi-monoubiquitination. Curiously though, lanes 2 and 3 did have a single band $\left(^{*}\right)$ corresponding to a monoubiquitinated $\alpha$-tubulin species (Figure 7c); a similar band was also evident when analyzed for endogenous ubiquitin (Figure $7 \mathrm{~b}$ ). These observations led us to believe that monoubiquitination of $\alpha$-tubulin occurred even in the absence of catalytic activity of MGRN1 (as in lanes 2 and 4), with enforced multi-monoubiquitination in the presence of MGRN1 $\Delta \mathrm{R}$ and $\mathrm{KO}$ (lane 4). Results similar to that obtained with Ub and MGRN1/MGRN1 $\Delta \mathrm{R}$ were observed when instead of $\mathrm{Ub}$, its mutant $\mathrm{K} 6$ was used (lanes 5 and 6). These data suggest that although MGRN1 promotes polyubiquitination, it also does not encourage multi-monoubiquitination of $\alpha$-tubulin.

No discernible change was detected in the ubiquitination pattern of $\beta$ - and $\gamma$-tubulins in the presence of the different HA-Ub constructs coexpressed with MGRN1 or MGRN1 $\Delta \mathrm{R}$ (Figures 7d and e).

The polyubiquitination of $\alpha$-tubulin in the presence of MGRN1, however, does not regulate its protein turnover. This was evident because of two reasons. First, K48-mediated polyubiquitination of $\alpha$-tubulin occurred irrespective of the expression of MGRN1 or MGRN1 $\Delta R$. (Supplementary Figure S6A). Similar argument also negates the role of K11, K29 and K63 ubiquitin residues in the polyubiquitin chain extensions (Supplementary Figure S6A). Second, treatment with the proteasomal inhibitor, MG132, similarly affects the S/P ratio of lysates from cells overexpressing MGRN1, MGRN1 $\Delta R$ or C316DMGRN1 (Supplementary Figure S6B), again stating that MGRN1 does not modulate $\alpha$-tubulin protein levels.

Furthermore, to establish the role of MGRN1-mediated $\alpha$-tubulin ubiquitination in endogenous MT polymerization, at $24 \mathrm{~h}$ after transfection, the cell lysates separated into cytosolic (soluble $(S)$ ) and insoluble (pellet $(P)$ ) fractions were biochemically analyzed to detect any change in $\alpha$-tubulin polymerization among cells expressing Ub, K0 or $\mathrm{K} 6$ along with MGRN1 or MGRN1 $\mathrm{R}$ (Figures $8 \mathrm{a}$ and $\mathrm{c}$ ). Our results showed that although the S/P ratio is $\sim 1$ in the presence of $\mathrm{Ub} / \mathrm{K} 6$ and MGRN1, this ratio is $>1.5$ in cells expressing $\mathrm{KO}$ and/or MGRN1 $\Delta \mathrm{R}$ (Figures $8 \mathrm{~b}$ and $\mathrm{d}$ ).

However, similar changes in the ubiquitination and polymerization pattern of $\beta$ - and $\gamma$-tubulins in the presence of the different HA-Ub constructs coexpressed with MGRN1 or MGRN1 $\Delta R$ could not be detected (Figures $8 \mathrm{e}-\mathrm{h}$ ).

These results so far taken together hint towards a unique mode of ubiquitin-mediated post-translational modification of $\alpha$-tubulin, where two ubiquitin E3 ligases act. An unknown ligase monoubiquitinates $\alpha$-tubulin and this happens even in the presence of MGRN1 $\Delta \mathrm{R}$. Either following this or independently, MGRN1 polyubiquitinates $\alpha$-tubulin via noncanonical K6 linkages as a post-translational modification to ensure proper polymerization but not for its degradation. In the absence of a catalytically active MGRN1, multi-monoubiquitination occurs that, however, is insufficient for complete $\alpha$-tubulin polymerization.

Such multiple E3 ligases working together and modifying a protein has been observed previously in case of some of the most important cell cycle regulators that in turn need to be under very tight regulation. The most studied protein to fall in this class is the tumor suppressor p53 (protein 53), known to be ubiquitylated by at least 11 different E3 ligases. ${ }^{34}$ Even though these E3 ligases have other targets, they all contribute individually in regulating p53 levels, which is vital for proper cell cycle progression.

Polyubiquitination of $\alpha$-tubulin affects spindle pole orientation. To assess the physiological consequence of MGRN1-mediated polyubiquitination of $\alpha$-tubulin, tilt in spindle poles was used as a read-out. HeLa cells were transfected with Ub or K0 along with MGRN1 or MGRN1 $\Delta \mathrm{R}$, and the mitotic cells scored for the tilt in the axis of cell division. The spindle angle for cells expressing MGRN1 and Ub was $\leq 10^{\circ}$ for $\sim 90 \%$ of cells (23 out of 27 cells; Figures $8 \mathrm{i}$ and $\mathrm{j}$ ). However, a tilt of $>10^{\circ}$ was observed in $\geq 55 \%$ of cells expressing MGRN1 and K0 or MGRN1 $\Delta \mathrm{R}$ and Ub, and $>10^{\circ}$ tilt was observed in $60 \%$ of cells expressing MGRN1 $\Delta \mathrm{R}$ along with $\mathrm{K} 0$. Thus, just skewing the ubiquitination balance from polyubiquitinated $\alpha$-tubulin to a more predominantly monoubiquitinated species was sufficient to cause spindle misorientation. Results similar to those with MGRN1 and Ub were also seen in the presence of MGRN1 and $\mathrm{K} 6$, where $\sim 70 \%$ of transfected cells ( 21 out of 30 cells) showed angle of tilt $\leq 10^{\circ}$, whereas $\sim 67 \%$ of cells (20 out of 30 cells) expressing $\mathrm{K} 6$ and MGRN1 $\mathrm{R}$ had $>10^{\circ}$ angle of tilt (Figures $8 \mathrm{i}$ and $\mathrm{j}$ ). Polyubiquitination of $\alpha$-tubulin by catalytically active MGRN1 was a crucial governing factor in the proper orientation of spindle poles.

\section{Discussion}

This study elucidates a novel interaction between the cytoskeletal protein $\alpha$-tubulin and MGRN1, whose absence leads to spongiform neurodegeneration or causes significant developmental defects during embryogenesis. The present study for the first time highlights how loss of the ubiquitin 
E3 ligase activity of MGRN1 affects spindle orientation. In cultured cell systems, MGRN1 physically associated with $\alpha$-tubulin, with an increased preference for polymerized MTs.
In cell lines and primary cells, functional inactivation of MGRN1 affected $\alpha$-tubulin polymerization. This also simultaneously coincided with an increase in the angle of tilt in

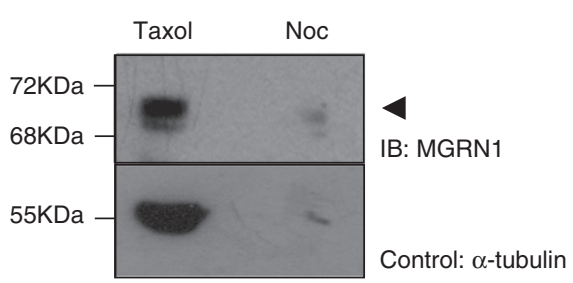

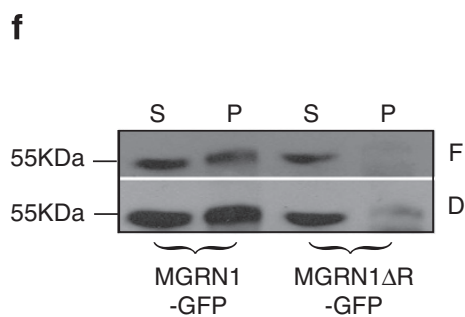

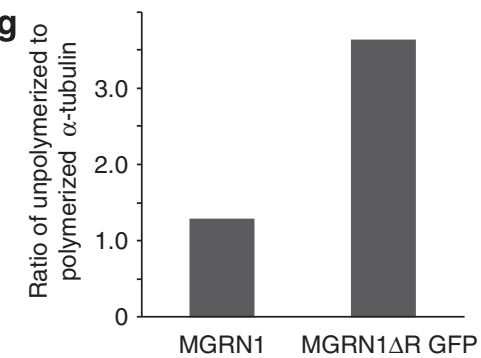

b
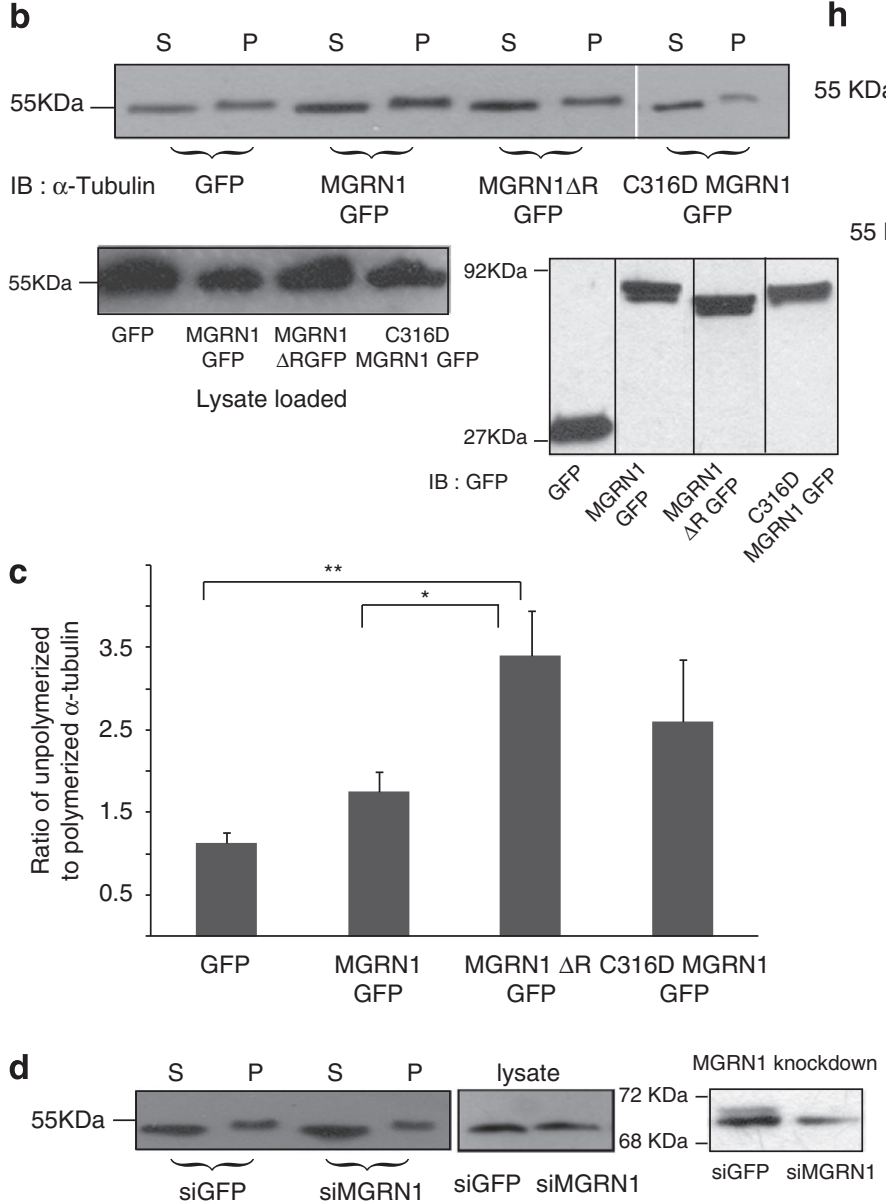

IB : $\alpha$-Tubulin

IB : Mgrn h

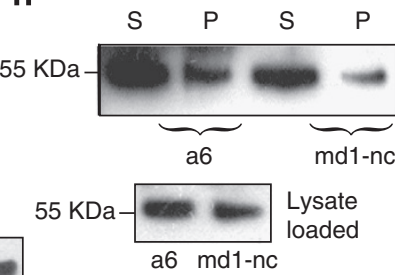

IB: $\alpha$-tubulin
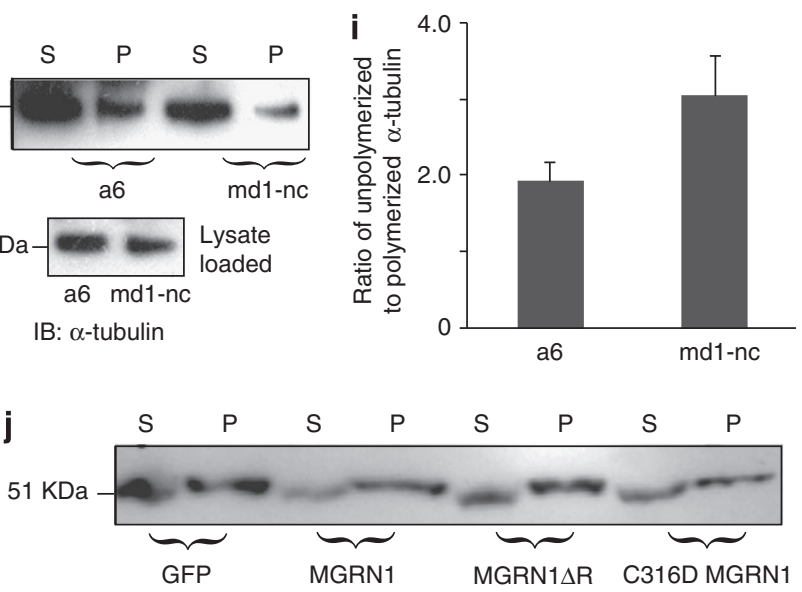

IB : $\beta$-Tubulin

GFP

GFP
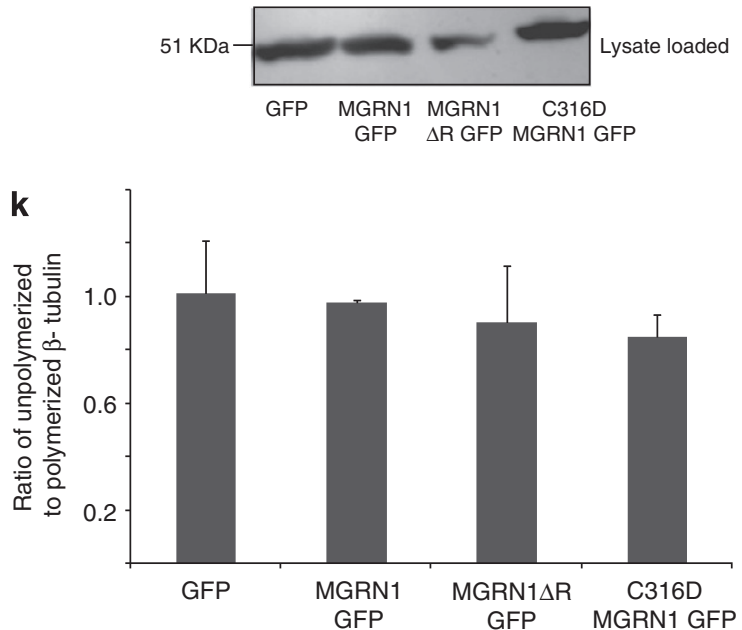

I

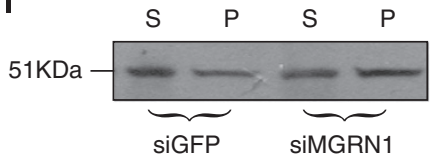

IB : $\beta$-Tubulin siGFP siMGRN1

$51 \mathrm{KDa}$

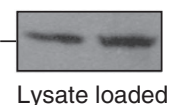

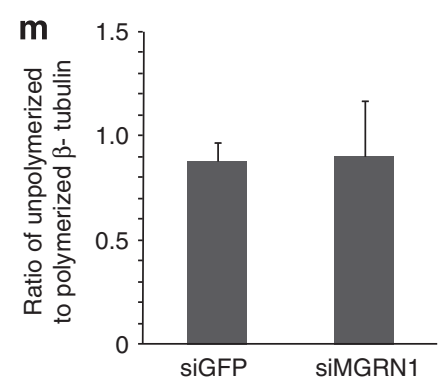




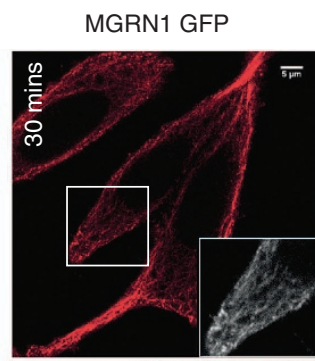

MGRN1 $\triangle$ R GFP
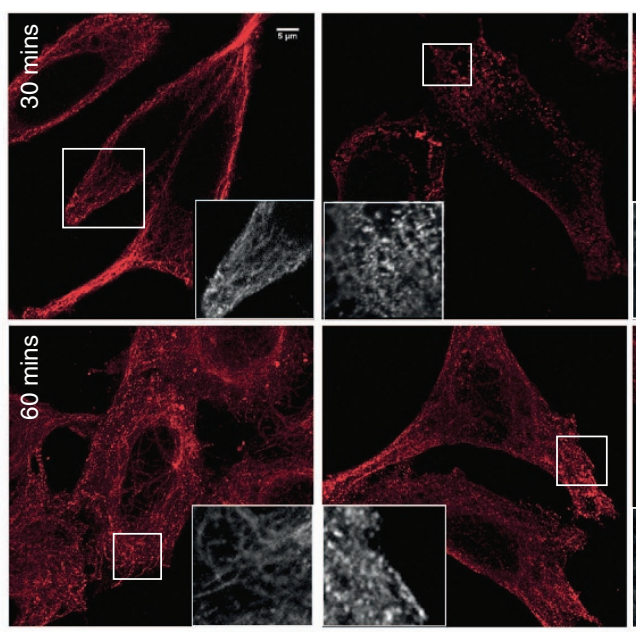

C316D MGRN1 GFP

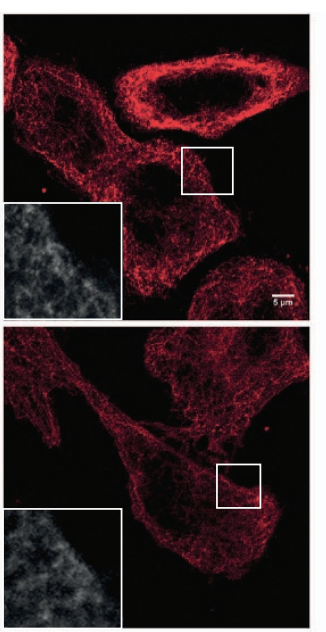

b $\alpha-$ Tubulin

MGRN1

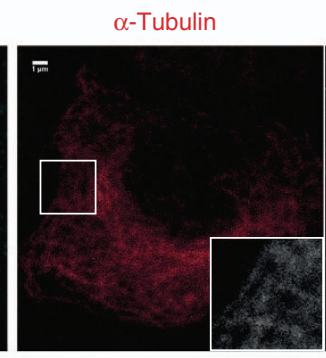

MGRN1
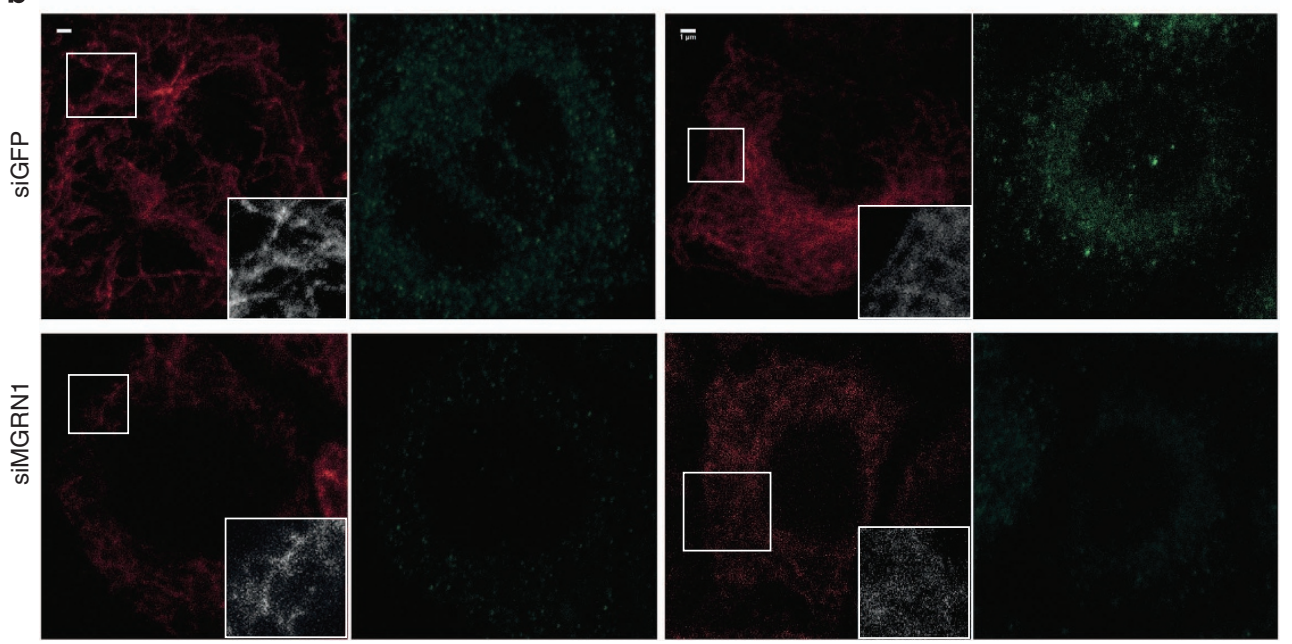

60 mins

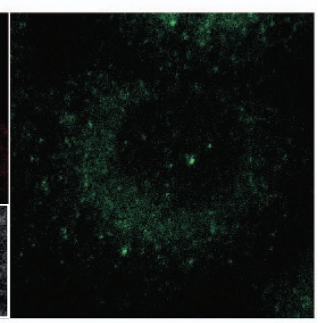

30 mins

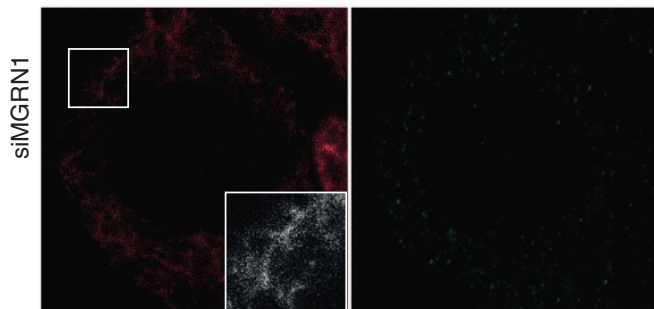

Figure 5 MGRN1 affects microtubule regrowth. (a) HeLa cells transiently transfected with the indicated constructs were treated with nocodazole, allowed to recover for 30 and $60 \mathrm{~min}$ and microtubule regrowth was monitored by immunostaining with $\alpha$-tubulin antibody. Insets of higher magnification show punctate $\alpha$-tubulin staining in the presence of MGRN1 $\triangle$ R contrary to a well-formed MT network in MGRN1-expressing cells. (b) HeLa cells treated with MGRN1 or GFP siRNAs were subjected to similar treatments as in (a), with recovery times of 30 and $60 \mathrm{~min}$. Insets of higher magnification show punctate $\alpha$-tubulin staining in the presence of MGRN1 siRNA, similar to MGRN1 $\Delta R$

\footnotetext{
Figure 4 MGRN1 affects polymerization of $\alpha$-tubulin. (a) Microtubule pulldown assay shows that MGRN1 co-pelleted with taxol-stabilized microtubules in mitotic HeLa cell lysates, indicated by ( $\mathbf{)}$. Nocodazole (Noc) inhibition of microtubule polymerization was used as negative control. The blot is a representative of at least three experiments. (b) Hela cells lysates, transiently expressing the indicated constructs, were fractionated to separate polymerized and unpolymerized fractions by high-speed ultracentrifugation; the fractionated samples along with total lysates were immunoblotted for $\alpha$-tubulin. The levels of $\alpha$-tubulin in the total lysates serve as loading control; the expression of the various constructs was biochemically analyzed with anti-GFP antibody. The blots are representative of at least five experiments. (c) The immunoblots generated in (b) were analyzed for the ratio of unpolymerized to polymerized $\alpha$-tubulin. Note an increase in this ratio upon functional depletion of MGRN1, an indication of a compromise in tubulin polymerization. ${ }^{*} P<0.05,{ }^{*} P<0.01$, using Student's $t$-test. Error bars, S.E.M. (d) HeLa cells treated with MGRN1 or GFP siRNAs were fractionated and immunoblotted as in (b). The blots are representative of at least three experiments. The inset immunoblot shows a efficient knockdown of MGRN1. (e) The immunoblots generated in (d) were analyzed for the ratio of unpolymerized to polymerized $\alpha$-tubulin. Note that MGRN1 siRNA treatment phenocopies expression of catalytically inactive MGRN1 expression. Error bars, S.E.M. (f) MEF cell lysates, transiently expressing the indicated constructs, were fractionated and immunoblotted as in (b). Note that faint (F) and dark (D) exposures of the blot show negligible amounts of detectable $\alpha$-tubulin in the pellet fraction with MGRN1 $\Delta R$. (g) The immunoblot generated in (f) was analyzed for the ratio of unpolymerized to polymerized $\alpha$-tubulin. Note that the functional depletion of MGRN1 has very similar effects on primary and HeLa cells. (h) Lysates from melanocytes, melan a-6 and md1-nc were fractionated into polymerized and unpolymerized fractions; these samples along with the total cell lysates were immunoblotted for $\alpha$-tubulin. The blot shown is representative from three experiments. (i) The immunoblots generated in (h) were analyzed for the ratio of unpolymerized to polymerized $\alpha$-tubulin. Note that this ratio in melan md1-nc (MGRN1 null cells) is very similar to that of MGRN1 knockdown in HeLa cells as shown in (d) and (e). Error bars, S.E.M. (j) The same samples analyzed in (b) were immunoblotted for $\beta$-tubulin. The levels of $\beta$-tubulin in the total lysates serve as loading control. The blots are representative of at least three experiments. (k) The immunoblots generated in (j) were analyzed for the ratio of unpolymerized to polymerized $\beta$-tubulin. Note no change in this ratio across samples. (I) The same samples analyzed in (d) were immunoblotted for $\beta$-tubulin. The levels of $\beta$-tubulin in the total lysates serve as loading control. The blots are representative of at least three experiments. (m) The immunoblots generated in (I) were analyzed for the ratio of unpolymerized to polymerized $\beta$-tubulin. Note no change in this ratio across samples
} 
a
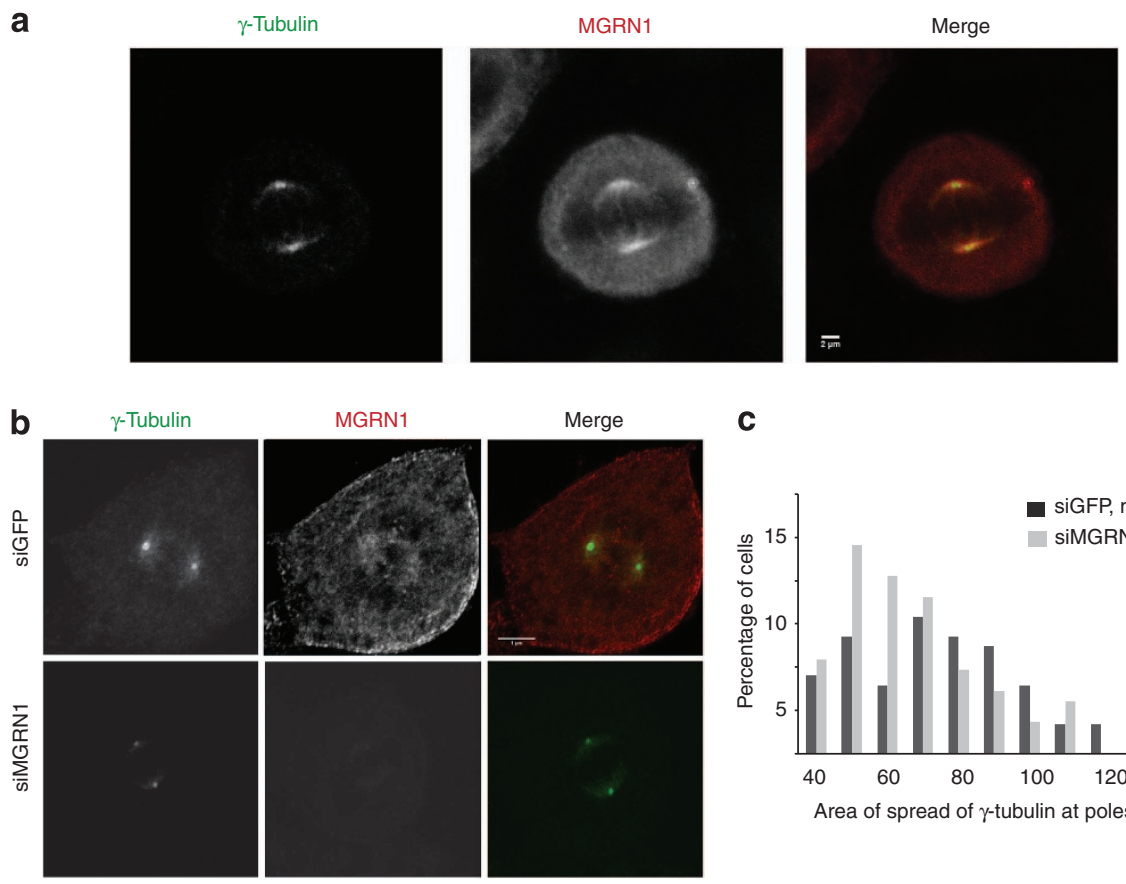

C

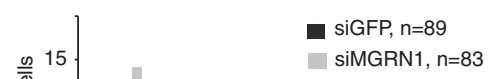

d

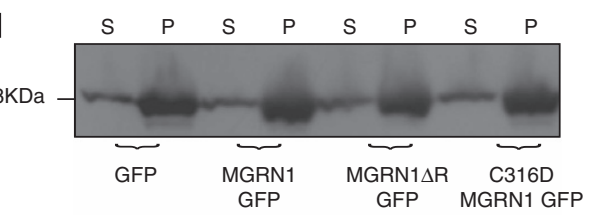

IB : $\gamma$-Tubulin

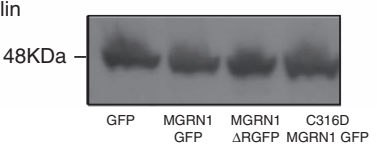

f

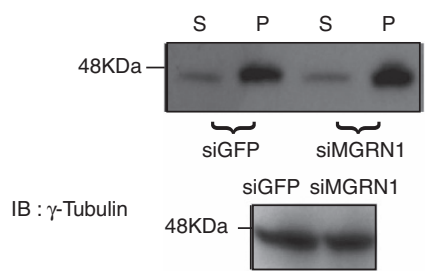

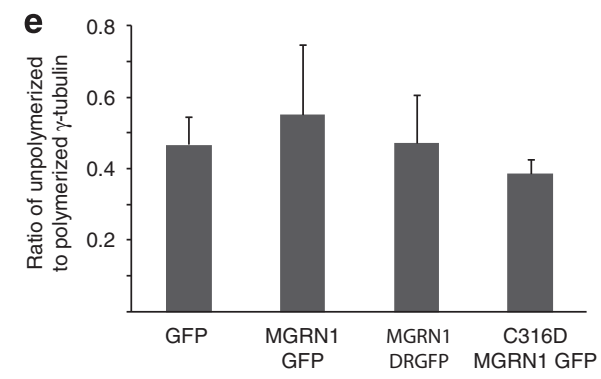

g

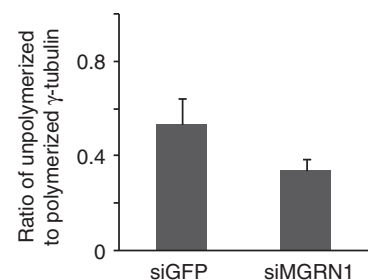

Figure 6 MGRN1 does not affect the spindle poles. (a) Asynchronous HeLa cells were co-immunostained for MGRN1 and $\gamma$-tubulin; mitotic cells were imaged. $\gamma$-Tubulin is detected at the spindle poles, whereas MGRN1 is seen to decorate the entire mitotic apparatus. (b) HeLa cells treated with MGRN1 or GFP siRNAs were co-immunostained for MGRN1 and $\gamma$-tubulin; mitotic cells were imaged. Similar $\gamma$-tubulin staining is detected at the spindle poles, irrespective of the treatments. (c) Cells imaged in (b) were analyzed for the spread of $\gamma$-tubulin staining at the spindle poles. Histogram plotting the percentage of cells at different of areas of spread as indicated on the $\mathrm{X}$ axis. Over 80 cells from 4 independent experiments were analyzed as represented for each of the siRNA treatments. (d) The same samples analyzed in Figure $4 \mathrm{~b}$ were immunoblotted for $\gamma$-tubulin. The levels of $\gamma$-tubulin in the total lysates serve as loading control. The blots are representative of at least three experiments. (e) The immunoblots generated in (d) were analyzed for the ratio of unpolymerized to polymerized $\gamma$-tubulin. Note no change in this ratio across samples. (f) The same samples analyzed in Figure $4 d$ were immunoblotted for $\gamma$-tubulin. The levels of $\gamma$-tubulin in the total lysates serve as loading control. The blots are representative of at least three experiments. (g) The immunoblots generated in (f) were analyzed for the ratio of unpolymerized to polymerized $\beta$-tubulin. Note no change in this ratio across samples

the axis of cell division, resulting in spindle misorientation. The effect of MGRN1 on spindle poles was specific for $\alpha$ tubulin as depletion of this protein did not affect either the expression pattern or polymerization status of $\beta$ - and $\gamma$ tubulins. Evidence for the importance of the E3 ligase activity of MGRN1 further came from the results that $\alpha$-tubulin polyubiquitination was achieved only in the presence of MGRN1 and Ub and not when MGRN1 $\Delta \mathrm{R}$ and/or K0 were expressed, suggesting that although MGRN1 promotes polyubiquitination, it also does not encourage multi-monoubiquitination of $\alpha$-tubulin. Monoubiquitination of $\alpha$-tubulin by an unknown E3 ligase and MGRN1-mediated polyubiquitination (utilizing the noncanonical K6 linkages) were most likely unrelated, independent events. However, modification of $\alpha$-tubulin by MGRN1 was crucial for proper spindle orientation. Accordingly, catalytic inactivation of MGRN1 
a

\begin{tabular}{|c|c|c|c|c|}
\hline $\begin{array}{l}\text { DNA Repair? } \\
\text { Protein degradation? }\end{array}$ & $\begin{array}{l}\text { Targeting to } 26 \mathrm{~S} \\
\text { proteasome }\end{array}$ & $\begin{array}{l}\text { DNA Repair? } \\
\text { Protein degradation? }\end{array}$ & $\begin{array}{l}\text { Targeting to } 26 \mathrm{~S} \\
\text { proteasome }\end{array}$ & $\begin{array}{l}\text { NF- } \kappa \text { B activation, } \\
\text { DNA repair andargeting tdysosome }\end{array}$ \\
\hline
\end{tabular}

b

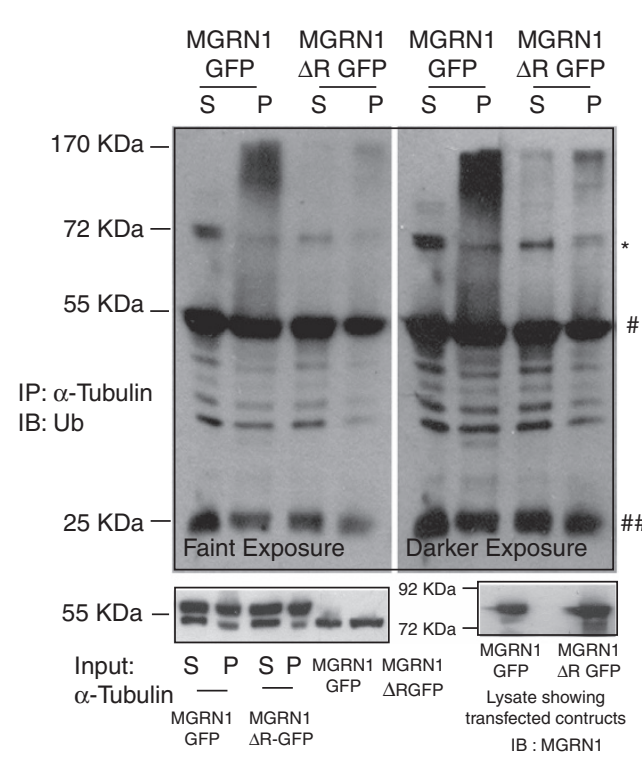

c

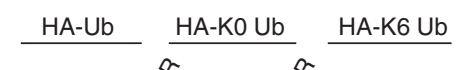

\section{西}

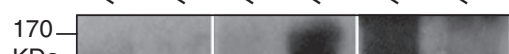

$\mathrm{KDa}$

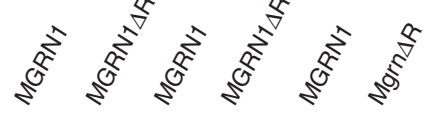

HA-Ub HA-KO Ub HA-K6 Ub

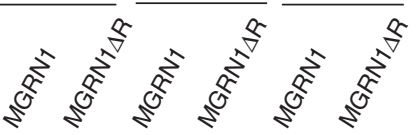

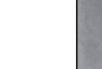

$72-$
$\mathrm{KDa}$

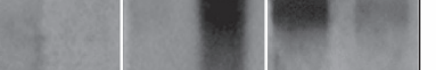

Da

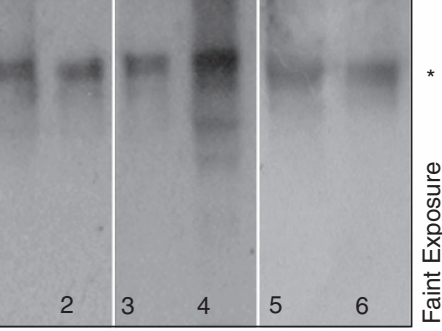

$55-$
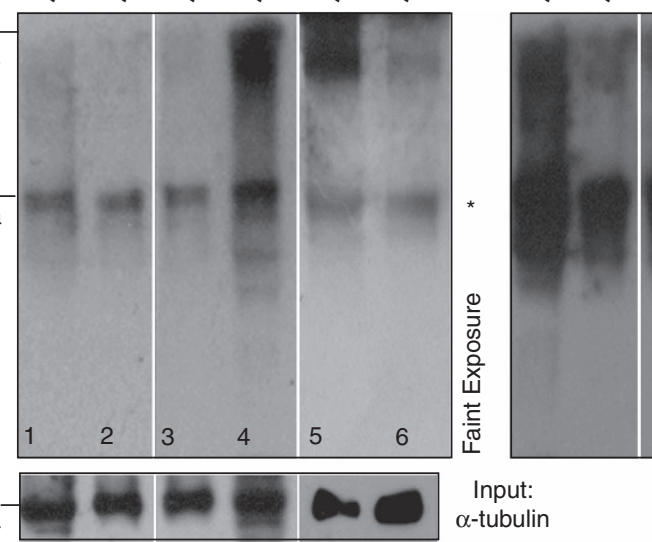

Input: $\alpha$-tubulin

IP: $\alpha$ tubulin

IB: HA d

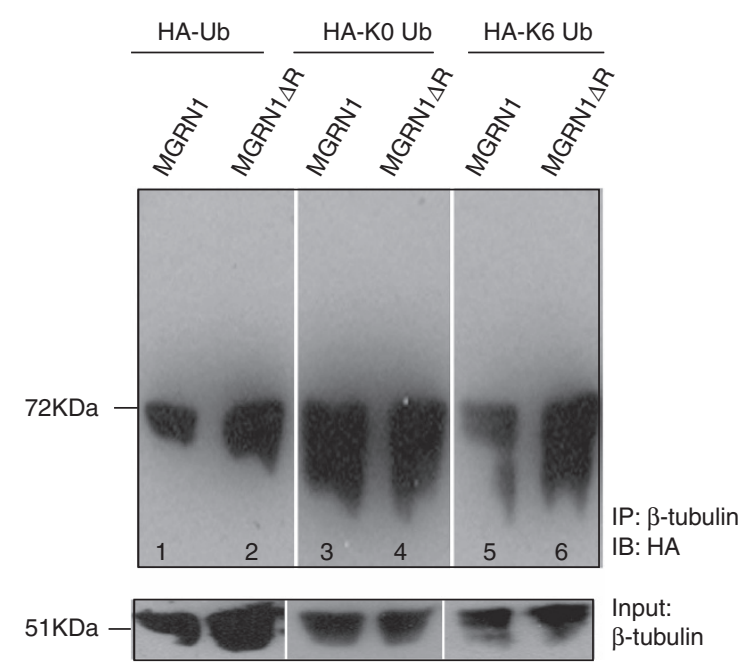

e

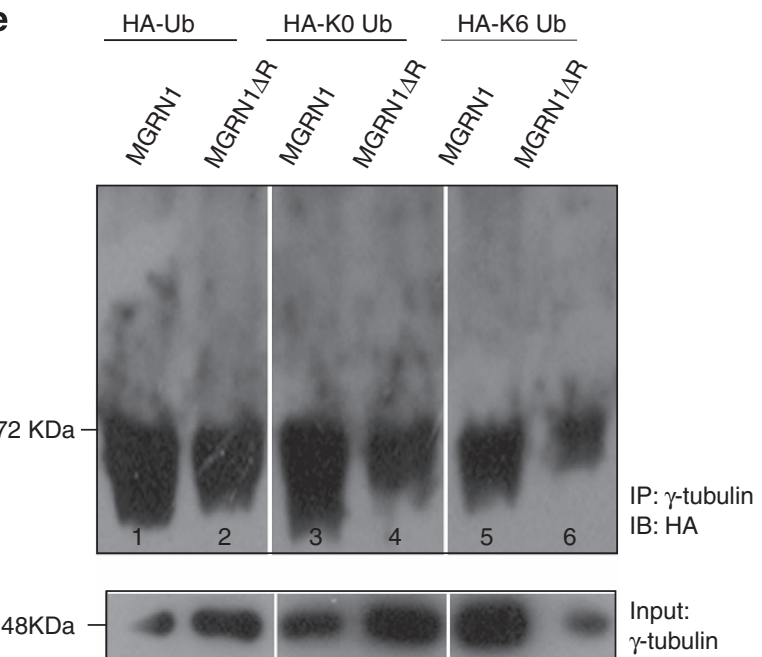

Figure 7 MGRN1 mediates $\alpha$-tubulin polyubiquitination. (a) A cartoon representation of the sequence of ubiquitin, the lysine residues involved in ubiquitination and their putative role in the cell. (b) HeLa cells transiently transfected with MGRN1-GFP or MGRN1 $\Delta$ R-GFP were fractionated to separate polymerized and unpolymerized fractions by high-speed ultracentrifugation, and the fractionated samples were immunoprecipitated with anti- $\alpha$-tubulin antibody. Enhanced in vivo ubiquitination was detected in pellet fractions compared with the supernatants by immunoblotting with anti-ubiquitin antibody. Faint and dark exposures of the anti-ubiquitin blot are shown. Expression of MGRN1 $\Delta R$ compromises the polyubiquitination in the pellet fraction. Expressions of $\alpha$-tubulin in the various fractions and total lysates along with that of MGRN1 in the total lysates serve as loading control. * Indicates monoubiquitinated $\alpha$-tubulin; " indicates immunoglobulin G IgG heavy chain; \#\# indicates IgG light chain. (c) HeLa cells transiently co-transfected with HA-Ub, HA-K0 or HA-K6 constructs along with MGRN1-GFP or MGRN1 $\Delta$ R-GFP were lysed and immunoprecipitated with anti $\alpha$-tubulin antibody. In vivo ubiquitination was detected by immunoblotting with anti-HA antibody. Faint and dark exposures of the anti-HA blot are shown. Polyubiquitination is detected in the presence of MGRN1 along with either Ub or K6. The input levels of $\alpha$-tubulin in the total lysates serve as loading control. (d) HeLa cells transiently co-transfected with HA-Ub, HA-K0 or HAK6 constructs along with MGRN1-GFP or MGRN1 $\triangle$ R-GFP were lysed, immunoprecipitated with anti $\beta$-tubulin antibody and immunoblotted with anti-HA antibody. The input levels of $\beta$-tubulin in the total lysates serve as loading control. (e) HeLa cells transiently co-transfected with HA-Ub, HA-KO or HA-K6 constructs along with MGRN1-GFP or MGRN1 $\triangle$ R-GFP were lysed, immunoprecipitated with anti $\gamma$-tubulin antibody and immunoblotted with anti-HA antibody. The input levels of $\gamma$-tubulin in the total lysates serve as loading control 
and consequential monoubiquitination of $\alpha$-tubulin resulted in misoriented mitotic spindle apparatus. We therefore conclude that ubiquitin-mediated post-translational modification of $\alpha$-tubulin that eventually affects its polymerization occurs via multiple E3 ligases, with MGRN1 being one of them (Figure 9). a
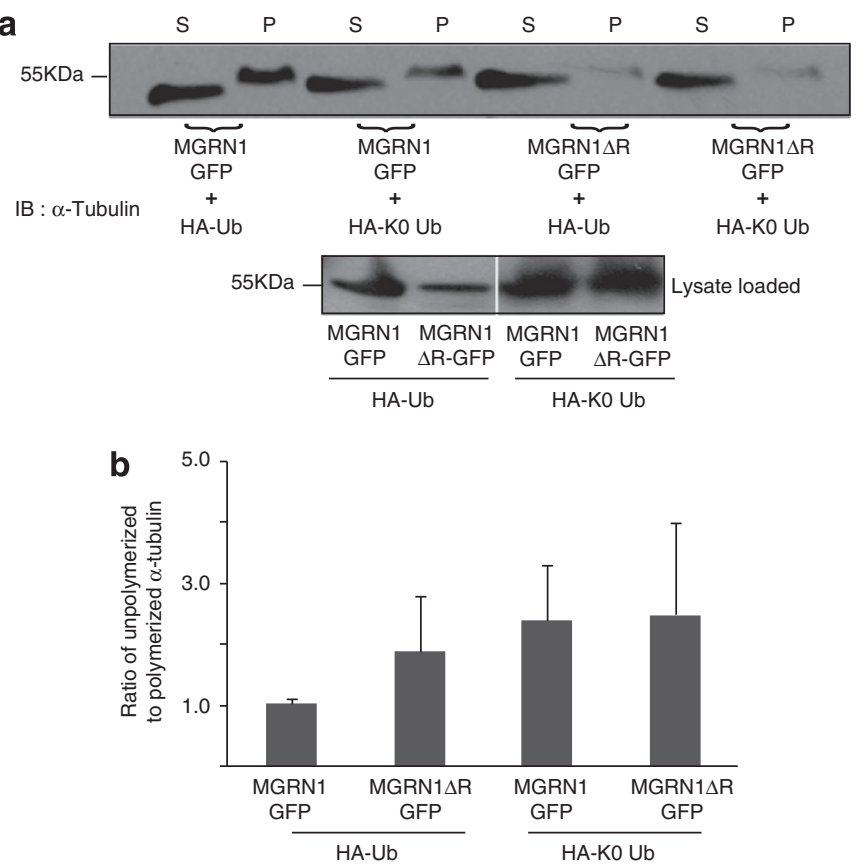

c

IB : $\alpha$-Tubulin

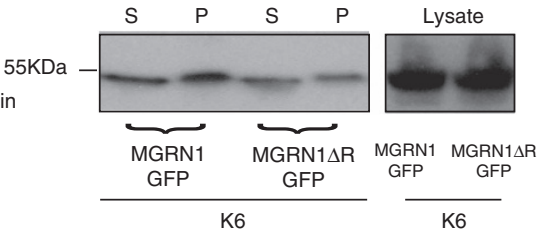

d

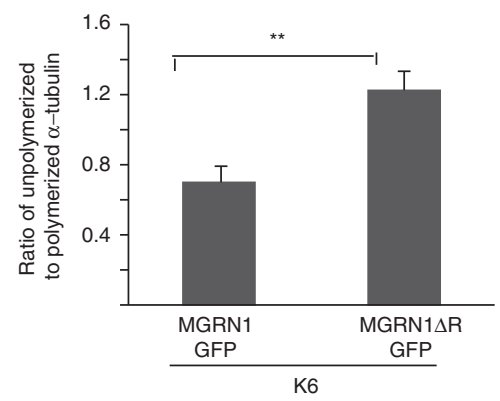

e

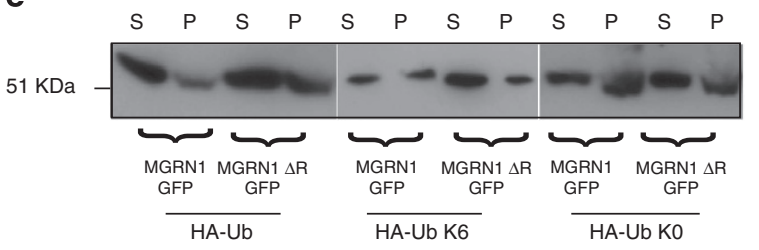

IB : $\beta$-Tubulin

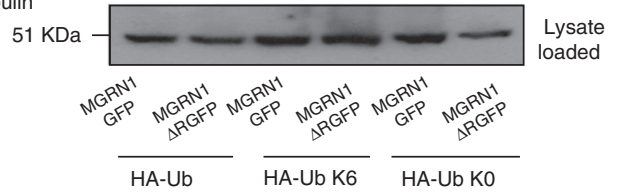

f

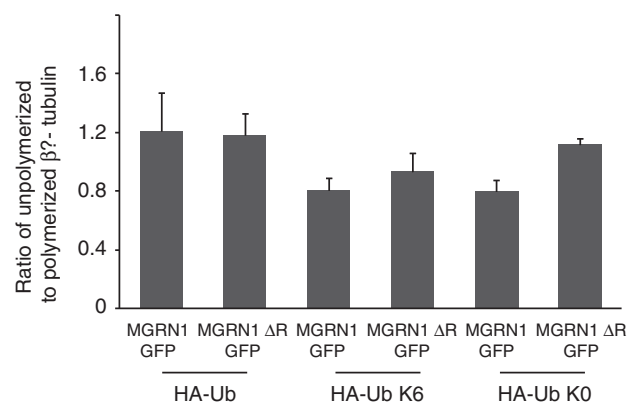

9

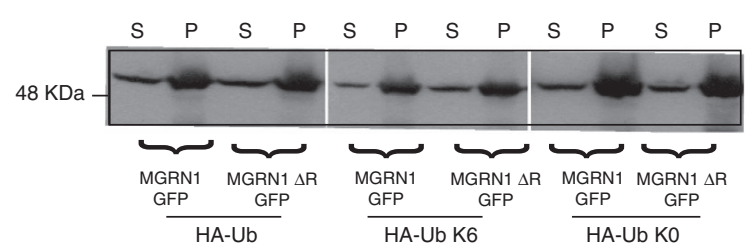

IB : $\gamma$-Tubulin

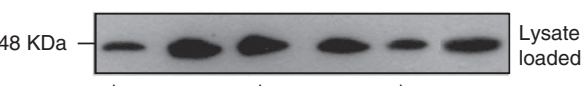

ysate

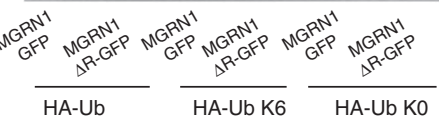

h
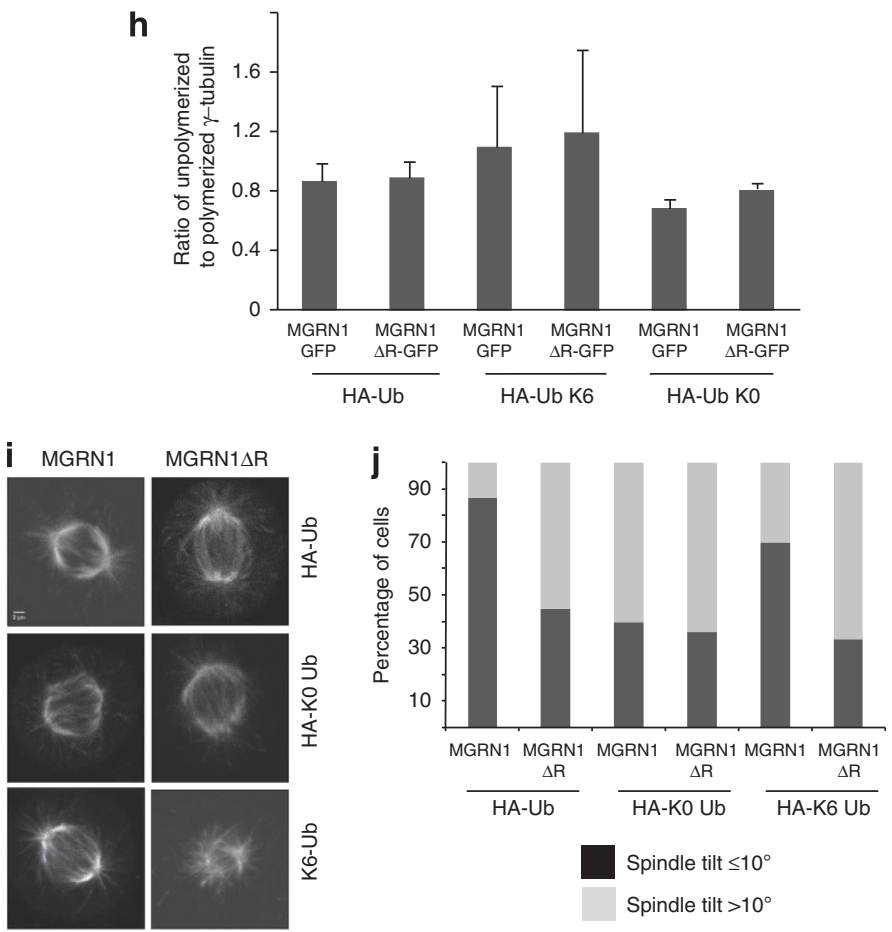
Vertebrates in general exhibit external bilateral symmetry; however, most internal organs such as the heart, lungs and kidney display asymmetry in structure and/or unilateral positioning with respect to the LR axis; interestingly, defects in laterality affects more than 1 in 8000 live births. ${ }^{35}$ Various models predict that the LR axis depends on either the presence of cilia-driven extracellular fluid flow ${ }^{36,37}$ or recruit 'ciliary' proteins, such as left-right dynein. ${ }^{38,39}$ as factors governing this asymmetry. However, a very recent report has shown in the plant model system (Arabidopsis thaliana) that mutations in $\alpha$-tubulin and in a $\gamma$-tubulin-associated protein (Tubgcp2) play an important role in the symmetry properties of the plant's axial organs. ${ }^{40,41}$ Similar mutations in vertebrate (Xenopus laevis), nematode (Caenorhabditis elegans) and mammalian cells affect the generation of LR asymmetry in a cilia-independent manner. ${ }^{42}$ Furthermore, expressions of mutant tubulins alter laterality by affecting proper localization of laterality related cargo molecules across the LR axis. ${ }^{42}$ This raised the possibility that alterations in $\alpha$-tubulin might as well have a drastic effect on the LR axis of symmetry.

A later study has further shown that mutations in $\beta$-tubulin disrupt spindle orientation while affecting MT dynamics, ${ }^{43}$ thus again emphasizing the importance of tubulins and their polymerization status in spindle orientation. However, this does not rule out the possibility that because of mutations, the association between the MT and the motor proteins may be compromised.

Although the association of tubulins with the motor proteins and hence their role cannot be undermined, the nature of the tubulin subunits themselves are also equally important for MT polymerization, stability and dynamics. Evidences already exist in varied experimental systems where changes in tubulins alter their polymerization status and MT stability, in turn playing a significant role in spindle orientation at the cellular level and the LR axis of symmetry at the organismal level. Hence, we hypothesize that instead of mutations in tubulin, a post-translational modification that would affect its polymerization status as is affected by the E3 ligase, MGRN1, would similarly alter $\alpha$-tubulin monomers and affect $\alpha$-tubulin functions at the cellular as well as the organismal levels.

When studying tubulin polymerization, MT stability and spindle orientation, the emphasis has been on various posttranslational modifications other than ubiquitination - the roles of PARKIN and BRCA1 as ubiquitin E3 ligases of $\alpha-/ \beta$ and $\gamma$-tubulins, respectively, have become evident only recently. ${ }^{9,11-13}$ It was, hence, plausible to speculate that several other E3 ligases might exist as tubulins are one of the fundamental proteins whose functions regulate a plethora of cellular activities and in turn would require stringent regulation by multiple modifiers. This would be similar to some of the most important cell cycle regulators (like p53, core histone proteins and Rev-erb $\alpha$ ) that are under very tight regulation by employing multiple E3 ligases. ${ }^{34,44,45}$

Although the ubiquitin molecule contains seven lysine residues in positions $6,11,27,29,33,48$ and 63 , the most studied have been homogenous polyubiquitin linkages involving residues 48 and $63 .{ }^{46,47}$ The involvement of the other lysine residues in regulating different cellular functions is progressively becoming evident ${ }^{45}-\mathrm{K} 6$ polyubiquitin chains being lesser known. The K6 chains have been observed in E3-independent reactions catalyzed by radiation gene 6 (Rad6), the yeast ortholog of $\mathrm{UbcH} 2$ (ubiquitin conjugating enzyme 2 (human)). ${ }^{48}$ More recently, evidences suggest that autoubiquitination of BRCA1 in vivo is mediated by $\mathrm{K} 6$ or K29 residues. ${ }^{32,49}$ Our present study identifying the polyubiquitination of $\alpha$-tubulin by MGRN1 preferentially utilizing $\mathrm{K} 6$, an unconventional site for ubiquitin polymerization, was unexpected. Although our results indicated that MGRN1mediated $\alpha$-tubulin ubiquitination did not involve K11, K29, $\mathrm{K} 48$ or $\mathrm{K} 63$ residues, it does not rule out the possibility of utilizing other lysine (K27, K33) residues or by linear chain extension.

Further insight into the mechanism of determination of the LR axis may come from a better understanding of MGRN1 function. Our results could place MGRN1 in the ubiquitous and essential pathway of ubiquitin-dependent post-translational modification of an indispensable cytoskeleton protein. However, its role in the modulation of MT would presumably be nonessential or functionally redundant, despite its rather widespread expression. Such functional redundancy would be essential for the regulation of a fundamental protein such as $\alpha$-tubulin, the disruption of which would affect not only cell division but also intracellular trafficking, cellular motility and morphology.

The study that identified the role of MGRN1 in embryonic patterning LR axis showed similar presence of Nodal expression among various MGRN1 mutant embryos. ${ }^{28} \mathrm{How}$ ever, the Nodal-responsive genes (Lefty1, Lefty2 and Pairedlike homeodomain transcription factor2) showed aberration in their expression patterns in the same samples, thus uncoupling the expression of Nodal from its responsive genes in the presence of MGRN1 mutants. In plants, tubulin

Figure 8 MGRN1 promotes $\alpha$-tubulin polymerization by polyubiquitination. (a) HeLa cells transfected with MGRN1-GFP or MGRN1 $\triangle$ R-GFP along with HA-Ub or HA-KO were fractionated and immunoblotted with $\alpha$-tubulin to check for the status of $\alpha$-tubulin polymerization. The levels of $\alpha$-tubulin in the total lysates serve as loading control. The blot is representative of at least three experiments. (b) The immunoblots from (a) were quantitated and analyzed for the ratio of unpolymerized to polymerized tubulin. Note that the functional MGRN1 and Ub are required for efficient $\alpha$-tubulin polymerization. Error bars, S.E.M. (c) HeLa cells transfected with MGRN1-GFP or MGRN1 $\Delta$ R-GFP along with HA-K6 were similarly treated as in (a). The levels of $\alpha$-tubulin in the total lysates serve as loading control.The blot is representative of at least three experiments. (d) Quantitation and analyses of immunoblots from (c) show that significantly more $\alpha$-tubulin exists in the polymerized state in the presence of MGRN1 and K6. Error bars, S.E.M. (e) The same samples generated in (a) and (c) were immunoblotted with $\beta$-tubulin. The levels of $\beta$-tubulin in the total lysates serve as loading control. The blot is representative of at least three experiments. (f) Quantitation and analyses of immunoblots from (e) show similar pattern of $\beta$-tubulin polymerization across samples. Error bars, S.E.M. (g) The same samples generated in (a) and (c) were immunoblotted with $\gamma$-tubulin. The levels of $\gamma$-tubulin in the total lysates serve as loading control. The blot is representative of at least three experiments. (h) Quantitation and analyses of immunoblots from (g) show similar pattern of $\gamma$-tubulin polymerization across different trasfections. Error bars, S.E.M. (i) HeLa cells transfected with MGRN1-GFP or MGRN1 $\triangle$ R-GFP along with HA-Ub, HA-K0 or HA-K6 were imaged and mitotic cells analyzed for the tilt in axis of division as in Figure 2. Note that conditions that support polyubiquitination (as in the presence of MGRN1 along with $\mathrm{Ub}$ or K6) only result in normal axis of cell division (with spindle tilt $\leq 10^{\circ}$ ). (j) The cells imaged in (i) were analyzed the amount of tilt in the axis of cell division was calculated similar to Figure 2. The graph shows the percentage of cells with abnormal tilt ( $>10^{\circ}$, in dark gray) and those with normal axis of division (tilt $\leq 10^{\circ}$, in light gray). Similar effect on the spindle angles was observed in the $\mathrm{Ub}$ and $\mathrm{K} 6$ 


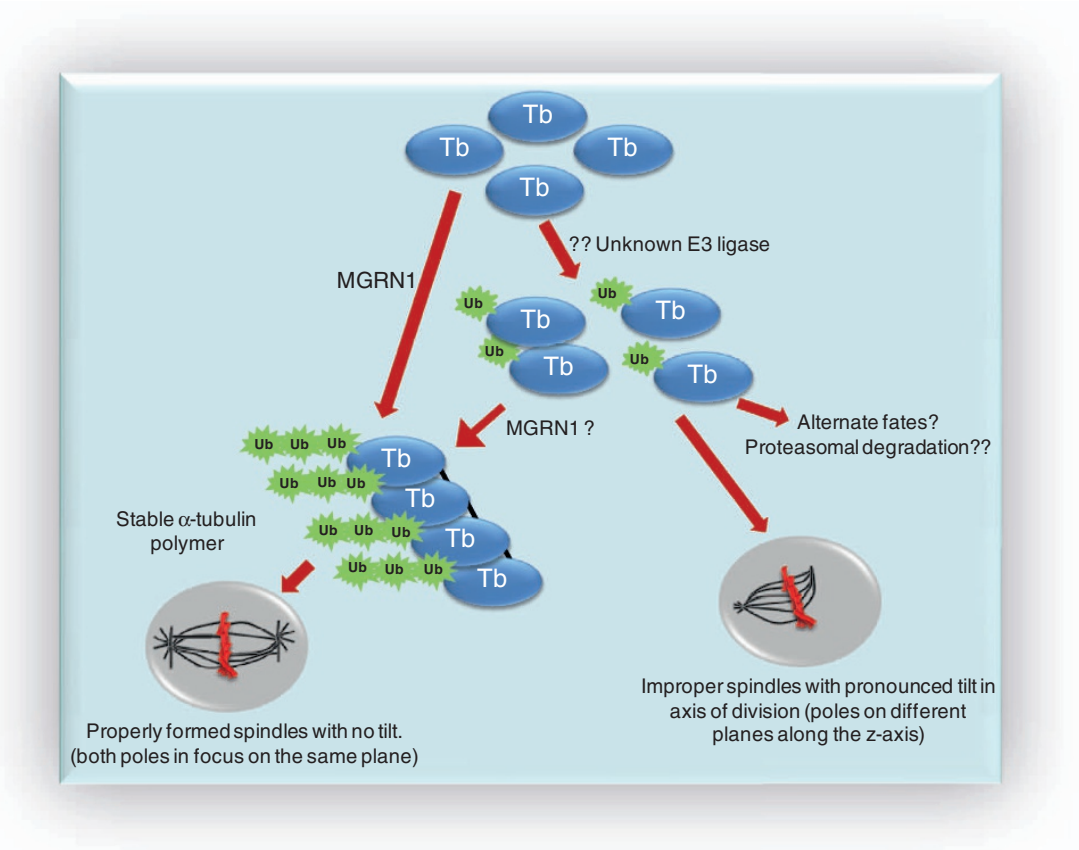

Figure 9 Schematic diagram summarizing the results. Tb denotes $\alpha$-tubulin. Although MGRN1-mediated polyubiquitination of $\alpha$-tubulin is crucial for its polymerization and proper orientation of the axis of cell division, its monoubiquitination by an unknown E3 ligase may be an independent event. Monoubiquitination of $\alpha$-tubulin preceeding MGRN1-mediated polyubiquitination also remains a possibility

mutations (spiral1, spiral2 and spira/3) produce right-handed helical growth; Lefty1 and Lefty2 mutants can act as suppressors of spiral1. ${ }^{40,41}$ However, in Xenopus, $\alpha$-tubulin mutations affecting the LR axis also alter Nodal expression pattern, ${ }^{42}$ hence elucidating that there is a close cross-talk and interplay between tubulins, Nodal and nodal-responsive genes; this need not follow a completely linear pathway.

The actual pathway by which MGRN1 alters nodalresponsive genes or helps orient the LR axis is yet under speculation; however, it would be prudent to extrapolate that MGRN1 through its post-translational modification of tubulin might alter the nodal-responsive genes, without affecting Nodal.

Finally, our study linking MGRN1 to the MT network via post-translational modification of $\alpha$-tubulin utilizing a more noncanonical $\mathrm{K} 6$ polyubiquitin linkage may highlight a key event in the LR patterning during early development in systems where stability of $\alpha$-tubulin subunits supersedes the association between the MT and the motor proteins during such developmental events.

\section{Materials and Methods}

Constructs and antibodies. MGRN1, MGRN1 $\Delta R$, MGRN1 $\Delta N$ and MGRN1 $\triangle C$ constructs have been described before. ${ }^{22}$ The C316DMGRN1 and C299EMGRN1 constructs were generated by standard site-directed mutagenesis techniques. HA-tagged wild-type Ub was a gift of Rafael Mattera (Bethesda, MD, USA); K0, K48 and K63 ubiquitin mutants were gifts of Kah-Leong Lim (Singapore); HA-tagged K6, K11 and K29 ubiquitin mutants were gifts of Tomohiko Ohta (Kawasaki, Japan). Antibodies were from the following sources: $\alpha$-tubulin (Santa Cruz Biotechnology, Dallas, TX, USA), $\beta$-tubulin (Abcam, Cambridge, UK), $\gamma$-tubulin (Sigma-Aldrich, St. Louis, MO, USA), dynein (Santa Cruz Biotechnology) and ubiquitin (Sigma-Aldrich). The MGRN1, GFP, RFP and HA antibodies were gifts of Ramanujan S Hegde (Cambridge, UK).
Cell culture, synchronization and immunocytochemistry. Cell lines used for the experiment were HeLa (human cervical cancer cell line), HEK 293T (human embryonic kidney cell line), MEF (mouse embryonic fibroblast cells), immortal melanocytes (control melan-a6 or Mgrn1-null mutations, melan md1-nc). ${ }^{27}$ Culture of HeLa, transient transfections, preparation of stable cell lines, immunofluorescent staining and fluorescence microscopy of fixed cells was as before. ${ }^{22,50}$ Briefly, cells were grown in $10 \%$ fetal bovine serum (FBS; Gibco, Grand Island, NY, USA)/Dulbecco's modified Eagle's medium (DMEM; Himedia, Mumbai, India) media at $37^{\circ} \mathrm{C}$ and $5 \% \mathrm{CO}_{2}$. At $\sim 90 \%$ confluency, cells were transfected with DNA using Lipofectamine 2000 (Invitrogen, Carlsbad, CA, USA) as per the manufacturer's instructions. At $\sim 24 \mathrm{~h}$ after transfection, cells were lysed using suitable lysis buffer. Immortal melanocytes were grown in $10 \%$ fetal calf serum (FCS; Gibco)/RPMI-1640 (Gibco)/200 nM 12-O-tetradecanoylphorbol 13-acetate (TPA; Sigma-Aldrich) media at $37^{\circ} \mathrm{C}$ and $10 \% \mathrm{CO}_{2}$, as per the guidelines of the Wellcome Trust Functional Genomics Cell Bank. HEK293T (gift of Subrata Banerjee, Kolkata, India) and MEF ((CF-1 strain) gift of Mitradas M Panicker, Bengaluru, India) cells were also grown under standard cell culture conditions. Immortal melanocytes (gift of Ramanujan S Hegde) were obtained from the Wellcome Trust Functional Genomics Cell Bank.

All tissue culture plasticware and Lab-Tek 8-well chambered slides used for microscopy were from Nunc, Rosklide, Denmark, and bottom coverglass dishes used for microscopy were from SPL Lifesciences, Gyeonggi-do, Korea. For synchronization of HeLa cells, thymidine- synchronization method was followed. Briefly, cells were subjected to $5 \mathrm{mM}$ thymidine (Sigma-Aldrich) treatment for 16$18 \mathrm{~h}$ followed by a release of $8 \mathrm{~h}$. This was followed by a Noc (Sigma-Aldrich) block at $100 \mathrm{ng} / \mathrm{ml}$ for $16 \mathrm{~h}$. The cells, now blocked at Gap2-mitosis (G2-M), were then allowed to enter mitosis for $60 \mathrm{~min}$ and then fixed (as in case of immunocytochemistry) or lysed (for biochemical studies).

Immunohistochemistry was done with minor modifications of earlier methods. ${ }^{22,50}$ For immunocytochemistry, cells were fixed with either $10 \%$ formaldehyde or methanol as per the requirement of the $\mathrm{Ab}$. Cells were permeabilized using $10 \% \mathrm{FBS} /$ phosphate buffer saline (PBS) $/ 0.1 \%$ saponin (Sigma-Aldrich) for $60 \mathrm{~min}$, followed by overnight staining in primary $\mathrm{Ab}$ at $4^{\circ} \mathrm{C}$ and $60 \mathrm{~min}$ of incubation in secondary $\mathrm{Ab}$ at room temperature. The samples were then imaged using confocal microscope.

Western blotting and immunoprecipitation. The protocol for western blotting was as before. ${ }^{22}$ Briefly, 10 or $12 \%$ tris-tricine gels were run as per the 
molecular weight being probed and then the proteins were transferred by wet electrophoretic transfer method for $55 \mathrm{~min}$ at $100 \mathrm{~V}$. For immunoprecipitation, the protocol was as described previously ${ }^{30}$ with antibodies as indicated in the figures. Briefly, at $24 \mathrm{~h}$ after transfection, cells were lysed in lysis buffer $(50 \mathrm{mM}$ Tris- $\mathrm{HCl}, \mathrm{pH} 7.5,150 \mathrm{mM} \mathrm{NaCl}, 0.1 \%$ Triton-X, 1\% IGEPAL, $1 \mathrm{mM}$ PMSF, protease inhibitor (Sigma-Aldrich)) and then immunoprecipitated using the standard protocol.

Separation of tubulin into polymerized and unpolymerized fractions. Whole-cell lysates were taken from $92 \mathrm{~mm}$ culture dishes with $1 \mathrm{ml}$ of lysis buffer ( $50 \mathrm{mM}$ HEPES, pH 7.4, $150 \mathrm{mM} \mathrm{NaCl}, 1 \% \mathrm{NP}-40,0.5 \%$ sodium deoxycholate, $0.1 \% \mathrm{SDS}$ ) at $4{ }^{\circ} \mathrm{C}$ for $20 \mathrm{~min}$. Soluble and insoluble fractions of cell lysates were separated by ultracentrifugation at 75000 r.p.m. for $1 \mathrm{~h}$ (rotor TLA 120.1, Beckman Coulter, CA, USA). A portion of the lysate (1/15th of the total) was saved before centrifugation. The soluble fraction $(S)$ and insoluble fraction $(P)$ were collected, and pellet was resuspended in Laemelli buffer ( $1 / 3$ volume of initial lysis buffer used). Equal volumes of supernatant and pellet fractions were then loaded for sodium dodecyl sulfate-polyacrylamide gel electrophoresis (SDS-PAGE), followed with analysis by western blotting.

FACS analysis. Cell cycle analysis was done on cells synchronized as before and labeled with $0.01 \mathrm{mM}$ propidium iodide (Thermo Scientific) at $37^{\circ} \mathrm{C}$ for $20 \mathrm{~min}$ Analysis was done on BD FACS Calibur flow cytometer (Becton Dickinson, San Jose, CA, USA)

Co-sedimentation assay. This was performed as described previously ${ }^{28}$ with minor modifications. Briefly, cells synchronized and enriched in mitotis were lysed at $4^{\circ} \mathrm{C}$ in $100 \mathrm{mM}$ 1,4-piperazinediethanesulfonic acid (PIPES), at pH 6.8, $1 \mathrm{mM} \mathrm{MgCl} 2,2 \mathrm{mM}$ ethylene glycol tetra acetic acid (EGTA) and 1\% Triton X-100, and spun at $13000 \times g$ for $30 \mathrm{~min}$. To the supernatant thus obtained, purified tubulin (Cytoskeleton, Denver, CO, USA; $4 \mu \mathrm{g})$, dithiothreitol $(1 \mathrm{mM})$, guanosine$5^{\prime}$-triphosphate (GTP; $\left.1 \mathrm{mM}\right)$ and taxol (Sigma-Aldrich) $(10 \mu \mathrm{M})$ were added to cleared lysates, and incubated for $1 \mathrm{~h}$ at $37^{\circ} \mathrm{C}$. Nocodazole $(100 \mu \mathrm{M})$ was added as a negative control. Lysates were layered over a $20 \%$ sucrose cushion in the above buffer and spun at 48000 r.p.m. for $1 \mathrm{~h}$ at room temperature (rotor TLA 120.1, Beckman Coulter). Microtubule pellets were collected after removing lysate and cushion, bound proteins were separated by SDS-PAGE and analyzed by western blotting.

Microtubule regrowth assay. At $20 \mathrm{~h}$ after transfection, microtubules were depolymerized in $10-20 \mu \mathrm{M}$ nocodazole in culture medium for $1 \mathrm{~h}$ at $37^{\circ} \mathrm{C}$. Cells were then washed and incubated in culture medium without nocodazole at $37^{\circ} \mathrm{C}$ to allow regrowth. Cells were fixed at different time intervals in 10\% formalin and processed for immunofluorescence microscopy to examine microtubule regrowth $(\alpha$-tubulin) from spindle poles in metaphase cells and the microtubule meshwork in interphase cells. To study the same in MEFs, the procedure was as described by Godin et al. ${ }^{7}$ Briefly, microtubules were depolymerized by treating cells with $5 \mathrm{mM}$ nocodazole for $1 \mathrm{~h}$ at $37^{\circ} \mathrm{C}$ and $30 \mathrm{~min}$ on ice. After treatment, cells were washed twice with $\mathrm{CO}_{2}$ equilibrated medium. Microtubules were allowed to regrow for different times (10 and $15 \mathrm{~min}$ ). This was followed by fixing and permebealization of cells as described above.

In vivo ubiquitination assay. In vivo ubiquitination assays were performed as described previously. ${ }^{30}$ Briefly, lysates of HeLa cells expressing the indicated GFP-tagged MGRN1, wild-type HA-tagged wild-type Ub or HA-tagged ubiquitin mutants (Ub-K0, Ub-K6, Ub-K11, Ub-K29, Ub-K48 and Ub-K63) were immunoprecipitated with various indicated anti-tubulin antibodies. Ubiquitinated tubulin was detected by immunoblotting with anti-HA antibodies. For analysis of ubiquitination of fractionationed samples, equal volumes of $S$ and $P$ (resuspended in same as initial volume of lysis buffer) were immunoprecipitated with $\alpha$-tubulin Ab and immunoblotted with ubiquitin $\mathrm{Ab}$.

Knockdowns with siRNA. ON-TARGETplus SMARTpool siRNAs against MGRN1 and GFP (catalog L-022620-00-0005 and D-001300-01-20; Thermo Scientific Dharmacon Products, Lafayette, CO, USA) were transfected using Lipofectamine 2000 following the manufacturer's instructions. Cells to be imaged were divided into two parts, trypsinized and replated - one set fixed $48 \mathrm{~h}$ after siRNA treatment, permeabilized and stained for immunocytochemistry, whereas another set lysed to check for knockdown efficiency biochemically.
Fluorescence microscopy and imaging. Fluorescence microscopy was performed utilizing LSM510-Meta and LSM710/ConfoCor 3 microscopy systems (Zeiss, Jena, Germany) equipped with an Ar-ion laser (for GFP excitation or Alexa-Flour 488 with the $488 \mathrm{~nm}$ line), a helium-neon (He-Ne) laser (for RFP, Alexa-Fluor 546 and 594 excitation with the 543 line) and a He-Ne laser (for AlexaFluor 633 with the 633 line). For all imaging, $63 \times 1.4$ numerical aperture (NA) oil immersion objective was used. For quantitative analyses and comparisons between multiple samples, images were collected using identical excitation and detection settings. The detector gain settings were chosen to allow imaging of the desired cells within the linear range of the photomultiplier tube without saturating pixels, unless otherwise specified.

Image analyses and calculation of spindle tilt. Image $(\mathrm{NIH}$, Bethesda, MD, USA) was used for all the image analyses reported in the text. For calculation of tilt in cells, $z$-stack images were taken with a $z$-spacing of $0.5-1.0 \mu \mathrm{m}$ and normalized as required. The distance between the poles was estimated by using ImageJ and then the spindle tilt was calculated as described by Delaval et al. ${ }^{28}$ The intensity or spread (i.e., area) was calculated using ImageJ. For analysis of long aster MTs, the length of aster rays was measured and the ratio of the length of aster ray to the cell diameter was determined. The central value of the ratio for control cells of 0.15 was taken as a cutoff mark. The number of cells with aster length to cell diameter ratio of $>0.15$ was calculated and plotted.

\section{Conflict of Interest}

The authors declare no conflict of interest.

Acknowledgements. We are grateful to R Mattera, K-L Lim and T Ohta for constructs, S Banerjee and MM Panicker for cells, R Mallik and A Manna for reagents, RS Hegde for cells and antibodies and Pallabi Bhattacharyya for generating C316DMGRN1 and C299EMGRN1 constructs. We thank P Majumder, R Mukherjee and Z Kaul for their valuable support and help throughout the study. This work was supported by the 'Integrative Biology on Omics Platform Project', intramural funding of the Department of Atomic Energy (DAE), Government of India.

1. Desai A, Mitchison TJ. Microtubule polymerization dynamics. Annu Rev Cell Dev Biol 1997; 13: 83-117.

2. Busson S, Dujardin D, Moreau A, Dompierre J, De Mey JR. Dynein and dynactin are localized to astral microtubules and at cortical sites in mitotic epithelial cells. Curr Biol 1998; 8: $541-544$.

3. Carminati JL, Stearns T. Microtubules orient the mitotic spindle in yeast through dyneindependent interactions with the cell cortex. J Cell Biol 1997; 138: 629-641.

4. O'Connell CB, Wang YL. Mammalian spindle orientation and position respond to changes in cell shape in a dynein-dependent fashion. Mol Biol Cell 2000; 11: 1765-1774.

5. Echeverri CJ, Paschal BM, Vaughan KT, Vallee RB. Molecular characterization of the $50-\mathrm{kD}$ subunit of dynactin reveals function for the complex in chromosome alignment and spindle organization during mitosis. J Cell Biol 1996; 132: 617-633.

6. Merdes A, Heald R, Samejima K, Earnshaw WC, Cleveland DW. Formation of spindle poles by dynein/dynactin-dependent transport of NuMA. J Cell Biol 2000; 149: 851-862.

7. Godin JD, Colombo K, Molina-Calavita M, Keryer G, Zala D, Charrin BC et al. Huntingtin is required for mitotic spindle orientation and mammalian neurogenesis. Neuron 2010; 67: 392-406.

8. Shimura H, Hattori N, Kubo S, Mizuno $\mathrm{Y}$, Asakawa $\mathrm{S}$, Minoshima $\mathrm{S}$ et al. Familial Parkinson disease gene product, parkin, is a ubiquitin-protein ligase. Nat Genet 2000; 25: 302-305

9. Ren $Y$, Zhao J, Feng J. Parkin binds to alpha/beta tubulin and increases their ubiquitination and degradation. J Neurosci 2003; 23: 3316-3324

10. Wloga D, Gaertig J. Post-translational modifications of microtubules. J Cell Sci 2010; 123 . 3447-3455.

11. Cuschieri L, Nguyen T, Vogel J. Control at the cell center: the role of spindle poles in cytoskeletal organization and cell cycle regulation. Cell Cycle 2007; 6: 2788-2794.

12. Parvin JD, Sankaran S. The BRCA1 E3 ubiquitin ligase controls centrosome dynamics. Cell Cycle 2006; 5 : 1946-1950.

13. Sankaran $\mathrm{S}$, Crone DE, Palazzo RE, Parvin JD. BRCA1 regulates gamma-tubulin binding to centrosomes. Cancer Biol Ther 2007; 6: 1853-1857.

14. Starita LM, Machida Y, Sankaran S, Elias JE, Griffin K, Schlegel BP et al. BRCA1dependent ubiquitination of gamma-tubulin regulates centrosome number. Mol Cell Biol 2004; 24: 8457-8466

15. Larsen $\mathrm{CN}$, Price JS, Wilkinson KD. Substrate binding and catalysis by ubiquitin C-terminal hydrolases: identification of two active site residues. Biochemistry 1996; 35: 6735-6744. 
16. Setsuie R, Wada K. The functions of UCH-L1 and its relation to neurodegenerative diseases. Neurochem Int 2007; 51: 105-111.

17. Betarbet R, Sherer TB, Greenamyre JT. Ubiquitin-proteasome system and Parkinson's diseases. Exp Neurol 2005; 191(Suppl 1): S17-S27.

18. Bheda A, Gullapalli A, Caplow M, Pagano JS, Shackelford J. Ubiquitin editing enzyme UCH L1 and microtubule dynamics: implication in mitosis. Cell Cycle 2010; 9: 9809-9894.

19. Day IN, Hinks LJ, Thompson RJ. The structure of the human gene encoding protein gene product 9.5 (PGP9.5), a neuron-specific ubiquitin C-terminal hydrolase. Biochem J 1990; 268: $521-524$.

20. Liu Y, Fallon L, Lashuel HA, Liu Z, Lansbury PT Jr. The UCH-L1 gene encodes two opposing enzymatic activities that affect alpha-synuclein degradation and Parkinson's disease susceptibility. Cell 2002; 111: 209-218.

21. Chakrabarti O, Ashok A, Hegde RS. Prion protein biosynthesis and its emerging role in neurodegeneration. Trends Biochem Sci 2009; 34: 287-295.

22. Chakrabarti $\mathrm{O}$, Hegde RS. Functional depletion of mahogunin by cytosolically exposed prion protein contributes to neurodegeneration. Cell 2009; 6: 1136-1147.

23. He L, Lu XY, Jolly AF, Eldridge AG, Watson SJ, Jackson PK et al. Spongiform degeneration in mahoganoid mutant mice. Science 2003; 299: 710-712.

24. Silvius D, Pitstick R, Ahn M, Meishery D, Oehler A, Barsh GS et al. Levels of the mahogunin ring finger $1 \mathrm{E} 3$ ubiquitin ligase do not influence prion disease. PLoS One 2013; 8: e55575.

25. Cota CD, Bagher P, Pelc P, Smith CO, Bodner CR, Gunn TM. Mice with mutations in Mahogunin ring finger-1 (MGRN1) exhibit abnormal patterning of the left-right axis. Dev Dyn 2006; 235: 3438-3447.

26. Jiao J, Kim HY, Liu RR, Hogan CA, Sun K, Tam LM et al. Transgenic analysis of the physiological functions of Mahogunin Ring Finger-1 isoforms. Genesis 2009; 47: 524-534.

27. Hida T, Wakamatsu K, Sviderskaya EV, Donkin AJ, Montoliu L, Lamoreux ML et al. Agouti protein, mahogunin, and attractin in pheomelanogenesis and melanoblast-like alteration of melanocytes: a cAMP-independent pathway. Pigment Cell Melanoma Res 2009; 22: 623-634.

28. Delaval B, Bright A, Lawson ND, Doxsey S. The cilia protein IFT88 is required for spindle orientation in mitosis. Nat Cell Biol 2011; 13: 461-468.

29. Caviston JP, Ross JL, Antony SM, Tokito M, Holzbaur EL. Huntingtin facilitates dynein dynactin-mediated vesicle transport. Proc Natl Acad Sci USA 2007; 104: 10045-10050.

30. Kim BY, Olzmann JA, Barsh GS, Chin LS, Li L. Spongiform neurodegeneration-associated E3 ligase Mahogunin ubiquitylates TSG101 and regulates endosomal trafficking. Mol Biol Cell 2007; 18: 1129-1142.

31. Mattera R, Puertollano R, Smith WJ, Bonifacino JS. The trihelical bundle subdomain of the GGA proteins interacts with multiple partners through overlapping but distinct sites. J Biol Chem 2004; 279: 31409-31418.

32. Tan JM, Wong ES, Kirkpatrick DS, Pletnikova O, Ko HS, Tay SP et al. Lysine 63-linked ubiquitination promotes the formation and autophagic clearance of protein inclusions associated with neurodegenerative diseases. Hum Mol Genet 2008; 17: 431-439.

33. Nishikawa H, Ooka S, Sato K, Arima K, Okamoto J, Klevit RE et al. Mass spectrometric and mutational analyses reveal Lys-6-linked polyubiquitin chains catalyzed by BRCA1-BARD1 ubiquitin ligase. J Biol Chem 2004; 279: 3916-3924.

34. Brooks CL, Gu W. p53 regulation by ubiquitin. FEBS Lett 2011; 585: 2803-2809.

35. Peeters H, Devriendt K. Human laterality disorders. Eur J Med Genet 2006; 49: 349-362.
36. Spéder P, Petzoldt A, Suzanne M, Noselli S. Strategies to establish left/right asymmetry in vertebrates and invertebrates. Curr Opin Genet Dev 2007; 17: 351-358.

37. Gros J, Feistel K, Viebahn C, Blum M, Tabin CJ. Cell movements at Hensen's node establish left/right asymmetric gene expression in the chick. Science 2009; 324 941-944.

38. Klar AJ. Support for the selective chromatid segregation hypothesis advanced for the mechanism of left-right body axis development in mice. Breast Dis 2008; 29: 47-56.

39. Levin M, Palmer AR. Left-right patterning from the inside out: widespread evidence for intracellular control. Bioessays 2007; 29: 271-287.

40. Abe T, Thitamadee S, Hashimoto T. Microtubule defects and cell morphogenesis in the lefty1lefty2 tubulin mutant of Arabidopsis thaliana. Plant Cell Physiol 2004; 45: 211-220.

41. Thitamadee S, Tuchihara K, Hashimoto T. Microtubule basis for left-handed helical growth in Arabidopsis. Nature 2002; 417: 193-196.

42. Lobikin M, Wang G, Xu J, Hsieh YW, Chuang CF, Lemire JM et al. Early, nonciliary role for microtubule proteins in left-right patterning is conserved across kingdoms. Proc Natl Acad Sci USA 2012; 109: 12586-12591.

43. Wright AJ, Hunter CP. Mutations in a $\beta$-tubulin disrupt spindle orientation and microtubule dynamics in the early Caenorhabditis elegans embryo. Mol Biol Cell 2003; 14: 4512-4525.

44. Singh RK, Gonzalez M, Kabbaj MH, Gunjan A. Novel E3 ubiquitin ligases that regulate histone protein levels in the budding yeast Saccharomyces cerevisiae. PLoS One 2012; 7 e36295.

45. Yin L, Joshi S, Wu N, Tong X, Lazar MA. E3 ligases Arf-bp1 and Pam mediate lithiumstimulated degradation of the circadian heme receptor Rev-erb alpha. Proc Natl Acad Sci USA 2010; 107: 11614-11619.

46. Pickart CM. Ubiquitin enters the new millennium. Mol Cell 2001; 8: 499-504.

47. Kravtsova-Ivantsiv Y, Ciechanover A. Non-canonical ubiquitin-based signals for proteasomal degradation. J Cell Sci 2012; 125: 539-548.

48. Baboshina OV, Haas AL. Novel multiubiquitin chain linkages catalyzed by the conjugating enzymes E2EPF and RAD6 are recognized by $26 \mathrm{~S}$ proteasome subunit 5. J Biol Chem 1996; 271: 2823-2831.

49. Wu-Baer F, Lagrazon K, Yuan W, Baer R. The BRCA1/BARD1 heterodimer assembles polyubiquitin chains through an unconventional linkage involving lysine residue $\mathrm{K} 6$ of ubiquitin. J Biol Chem 2003; 37: 34743-34746.

50. Rane NS, Kang SW, Chakrabarti O, Feigenbaum L, Hegde RS. Reduced translocation of nascent prion protein during ER stress contributes to neurodegeneration. Dev Cell 2008; 15: $359-370$

(i) (E) $€$ Cell Death and Disease is an open-access journal published by Nature Publishing Group. This work is licensed under a Creative Commons Attribution-NonCommercialNoDerivs 3.0 Unported License. To view a copy of this license, visit http://creativecommons.org/licenses/by-nc-nd/3.0/

Supplementary Information accompanies this paper on Cell Death and Disease website (http://www.nature.com/cddis) 